\title{
Natural and artificial atoms for quantum computation
}

\author{
Iulia Buluta ${ }^{1}$, Sahel Ashhab ${ }^{1,2}$, Franco Nori ${ }^{1,2}$ \\ ${ }^{1}$ Advanced Science Institute, RIKEN, Wako-shi, Saitama, 351-0198, Japan \\ ${ }^{2}$ Department of Physics, The University of Michigan, Ann Arbor, Michigan \\ 48109-1040, USA
}

\begin{abstract}
Remarkable progress towards realizing quantum computation has been achieved using natural and artificial atoms as qubits. This article presents a brief overview of the current status of different types of qubits. On the one hand, natural atoms (such as neutral atoms and ions) have long coherence times, and could be stored in large arrays, providing ideal "quantum memories". On the other hand, artificial atoms (such as superconducting circuits or semiconductor quantum dots) have the advantage of custom-designed features and could be used as "quantum processing units". Natural and artificial atoms can be coupled with each other and can also be interfaced with photons for long-distance communications. Hybrid devices made of natural/artificial atoms and photons may provide the next-generation design for quantum computers.
\end{abstract}

PACS numbers: 03.67.Lx 


\section{Introduction}

The experimental realization of Quantum Computation (QC) has been a challenge for more than a decade. While a fully operational quantum computer that could factorize thousand-digit numbers is still a distant goal, with the new technologies for the coherent manipulation of atoms, photons, and electrons, nowadays applications like quantum cryptography and quantum communication are already commercially available. Since potential QC implementations come in many shapes and sizes, it is difficult to quantify the overall progress in the field of QC. In order to assess the current state of the art in $\mathrm{QC}$, a comparison between the various approaches is needed. However, because these approaches are very different (in terms of the underlying physical processes, experimental techniques, and how well the physical system is understood), we should be careful not to compare apples with oranges. We would rather like to compare apples with apples, or in our case, atoms with atoms. Therefore, in this paper we consider natural and artificial atoms for implementing QC.

Among the most successful and rapidly developing ways of realizing QC are those using natural atoms (such as neutral atoms [1] or ions [2]) and artificial atoms (such as superconducting circuits [3, 4] or spins in solids [5]). Contrasting natural and artificial atoms would help highlighting their strengths. For the sake of comprehensiveness other QC approaches (i.e., with nuclear spins in molecules [6, 7] or in phosphorus impurities in silicon [8, 9], photons [10, 11], and so on) are also be briefly covered here. A complementary overview on qubits can be found in [12]. Although there are many exciting theoretical proposals, we will focus more on what has already been experimentally demonstrated and less on what could eventually be achieved in each system. We should stress from the beginning that our purpose is not to show that a certain system is better than others, but to review the current experimental state of the art in QC. One should keep in mind that some approaches are more recent than others, some benefit from technologies that have been developed before, while others had to develop their own new technologies on the way, and, most importantly, each approach has to deal with specific issues whose difficulty cannot be compared.

By considering natural and artificial atoms and their potential for implementing QC, we hope to gain a broader perspective of the current status of QC. Moreover, this approach may also provide a glimpse into the future of QC. However, we would rather not attempt to make any prediction regarding what system would be best for realizing a practical quantum computer. Ten or twenty years from now such speculation might sound as amusing as the prediction made by Popular mechanics in 1949: "In the future, computers may weigh no more than 1.5 tonnes."

After summarizing the characteristics of each system we discuss the strengths and weaknesses of natural and artificial atoms. Next, we take a look at hybrid systems and photon interfaces, and, finally, consider future prospects. The main issues discussed throughout the paper are collected in the six tables, which can be found at the end of the paper. For the reader interested in the details for a particular system, the Appendix 
provides extended tables. The list of references at the end tries to cover some of the recent experimental progress in the coherent control of natural and artificial atoms.

\section{Neutral atoms}

When looking for a physical system to realize qubits (which are controllable two-level systems), perhaps the most obvious candidate is neutral atoms [13, 14, 15, 16, 17, 18, 19, 20, 21, 22, 23, 24, 25, 26, 27, 28, 29, 30, 31, 32, 33, 34, 35, 36, 37, 38. Atoms have many energy levels that have been studied extensively over the past century, and some of these energy levels are extremely stable. Indeed, with accuracies better than one part in $10^{-15}$, atomic clocks provide the best available time and frequency standards. The qubits encoded in the atomic energy levels can be initialized by optical pumping and laser cooling, manipulated with electromagnetic radiation, and then measured via laser-induced fluorescence. In short, atoms provide clean, well-defined qubits (see also Box 2 (a,b) and Table A1).

Neutral atoms make attractive qubit candidates also because of their weak interaction with the environment, leading to long coherence times [15, 14, 19, 30]. They can be cooled down to nK temperatures and trapped in very large numbers (millions) in microscopic arrays created by laser beams (called optical lattices). The trapping and manipulation of atoms can be done with high precision [14, 18, 19, 21]. Until recently, the individual manipulation and measurement of neutral atoms in optical lattices was not possible, but the experiments in [24, 29, 35, 32, 31] show very promising perspectives for individual addressing and readout.

While one-qubit gates can be implemented with very high fidelity [34], realizing twoqubit gates or many-qubit entangled states is challenging because the atoms interact very weakly with each other. This problem can be overcome in several ways. For instance, the atoms can first be brought into a superposition of two internal spin states. Then, as the spin-dependent lattice is moved, the atoms go to the left and to the right simultaneously colliding with their neighbors. In this way, in a single operation a highly entangled many-qubit state can be created [13]. Unfortunately, these collisional gates are very sensitive to decoherence and are also quite slow 11. Exchange interactions provide an alternative approach [20, 22, 25]. The effective spin-spin interaction between two atoms in a double-well potential was used to demonstrate a two-qubit SWAP gate [20]. Furthermore, with polar molecules [17] or Rydberg atoms [28, 27, 36] dipole-dipole interactions could be exploited for realizing two-qubit gates. Very recently, a CNOT gate [33], post-selective entanglement of two atoms [37] using Rydberg blockade interactions and on-demand entanglement 38] have been demonstrated.

The prospect of producing many-qubit entangled states together with the possibility of single-site addressing and measurement make neutral atoms promising for the quantum simulation of condensed-matter physics [16, 23] as well as measurement-based QC [39]. 


\section{Ions}

While neutral atoms interact weakly among themselves, ions, being charged, interact rather strongly via Coulomb repulsion. This facilitates the implementation of twoqubits gates without compromising the long coherence times [40, 41, 42, 43, 44, 45, 46, 47, 48, 49, 50, 51, 52, 53, 54, 55, 56, 57, 58, 59, 60, 61, 62, 63, 64. Also thanks to their charge, the motion and position of the ions can be well controlled. Ions can be trapped by electrical (or magnetic) fields, laser-cooled and manipulated with high precision [2]. Quantum information can be encoded either in the internal (hyperfine or Zeeman sublevels, or the ground and excited states of an optical transition), or in the motional states (the collective motion of the ions). While the internal states exhibit very long coherence times (hyperfine transitions $>20 \mathrm{~s}$ [50] and optical transitions $>1 \mathrm{~s}$ ) the motional states have typical lifetimes of $<100 \mathrm{~ms}$. As in the case of neutral atoms, the initialization of the qubits can be done by optical pumping and laser cooling, and they can be measured with very high accuracy [59, 62] via laser-induced fluorescence. Scaling the current experiments to large numbers of ions is theoretically possible, but technically challenging. The proposed approaches to scalability include ion shuttling, two-dimensional ion arrays, photon interconnections, long equally-spaced strings, and two-dimensional Coulomb crystals (see [57] and Box 2 (c,d) and Table A2).

Using the collective motion of the ions as data bus, high-fidelity one-, two- [53, 56], and even three-qubit [60] gates have been experimentally demonstrated. Entangled (Greenberger-Horne-Zeilinger (GHZ) and W) states of up to 14 qubits have been realized [51, 52, 64]. Two-qubit gates can also be implemented using bichromatic excitation fields that produce coherent two-qubit transitions [42, 56] or by the stateselective displacement of the ions with an optical "pushing" force [41]. In the latter, the displacement changes the strength of the Coulomb repulsion, leading to an additional phase, so realizing a controlled-phase gate. Recently, a trapped ion quantum processor implementing arbitrary unitary transformations on two qubits has been realized [58].

Besides the generation of GHZ and W entangled states, quantum algorithms [44, 49], quantum teleportation [46, 48], entanglement of distant qubits [55], quantum error correction [47] and decoherence free qubits [61] have also been demonstrated with trapped ion qubits.

\section{Superconducting circuits}

Superconducting circuits [65, 66, 67, 68, 69, 70, 71, 72, 173, 74, 75, 76, 77, 78, 79, 80, 81, 82, 83, 84, 85, 86, 87, 88, 89, 90, 91, 92, 93, 94, 95, 96, 97, 98, 99, 100] are typically $\mu \mathrm{m}$-scale circuits operated at $\mathrm{mK}$ temperatures. Although macroscopic, they can still exhibit quantum behavior, which can be harnessed for QC [101, 4, 3, 102]. Superconducting circuits are $R L C$ circuits that also include nonlinear elements, called Josephson junctions. Thanks to superconductivity, the resistance vanishes $(R=0)$, eliminating the most serious source of dissipation and noise. Now, the $L C$ circuit is a 
harmonic oscillator. The problem with harmonic oscillators is that they have an infinite number of equally-spaced energy levels and therefore it is not possible to target only the lowest two energy levels. By introducing nonlinearity through the Josephson junction, the energy-level separation becomes nonuniform, and the lowest two levels can be used to encode the qubit [4, 3] (see also Box 1). Quantum information can be encoded in different ways: in the number of superconducting electrons on a small island (charge qubit), in the direction of a current around a loop (flux qubit), or in oscillatory states of the circuit (phase qubit). These qubits can be controlled by microwaves, voltages, magnetic fields, and currents as well as measured with high accuracy [84] using integrated on-chip instruments. The characteristics of the qubits can be designed and many qubits could be coupled in arrays. Therefore, superconducting qubits are flexible and promise the realization of QC on a chip (see Box 2 (e,f) and Table A4).

Superconducting qubits have coherence times that can reach tens of $\mu$ s (e.g., [98]), the coupling between qubits can be made strong and can be turned on and off electronically [74, 81]. In addition to direct coupling strategies, superconducting circuits can be coupled via "cavities" [83, 80, which are actually electrical resonators (and the "photons" are actually electron-density oscillations). This setup is promising for the study of circuit cavity Quantum Electrodynamics (circuit QED) [72, 47, 4, 3, 86].

With superconducting circuits one can now realize simple algorithms [88], and generate entangled states of three qubits [90, 91, 92] and arbitrary photon states in a resonator [103]. Other recent advances include the performance of quantum nondemolition measurements [79], the realization of multi-level quantum systems [99, 104], the violation of Bell's inequality [87, 95], and the coupling of a mechanical resonator to a superconducting qubit [94].

\section{Spins in solids}

Coherent control and measurement of single spins in solids [105, 106, 107, 108, 109, 110, 111, 112, 113, 114, 115, 116, 117, 118, 119, 120, 121, 122, 123, 9, 124, 125, 126, 58, 127, 128, 129, 130, 131, 132] is now possible, and this allows using electron spins in semiconductor quantum dots [115], or electron spins together with nuclear spins in nitrogen-vacancy (NV) color centers in diamond [114] for QC purposes [105, 5] (see Box $2(\mathrm{~g}, \mathrm{~h})$ and Table A5 which attempts to cover, as much as possible in such a short space, several very different systems under the broad umbrella of spins).

Quantum dots are nanoscale structures in which electrons are trapped in all three dimensions. They can be fabricated in several ways, for example, by growth or with electrode gates in a two-dimensional electron-gas. The material of choice is usually

GaAs. On the other hand, NV centers are point defects in the diamond lattice, consisting of a nearest-neighbor pair made of a nitrogen atom, substituting a carbon atom, and a lattice vacancy. Although in its early stages, quantum computing with electronic and nuclear spins in an array of phosphorus donor atoms embedded in a pure silicon lattice (P:Si) has recently achieved very encouraging results [133, 134, 135, 136, 137]. 
Solid state qubits such as quantum dots are attractive because, like superconducting circuits, they could be designed to have certain characteristics and assembled in large arrays. Furthermore, they require temperatures of up to a few K (NV centers in diamond could operate even at room temperature). The manipulation and readout can be done both electrically [117] and optically [118, 116, 122.

While Rabi oscillations have already been observed [112, 120], two-qubit gates have only been demonstrated for NV centers in diamond [108] (although, a SWAP gate between logical states has been realized [109]). However, long coherence times [119, 121] have been measured for both quantum dots $(\sim \mu \mathrm{s})$ [126, 129, 132, 125, 128] and NV centers $(>5 \mathrm{~ms})$ [123]. Moreover, for NV centers the entanglement between the electron and nuclear spins has also been shown [123].

Nowadays, Nuclear Magnetic Resonance (NMR) techniques are extensively used in the context of nuclear spins in semiconductors. NMR techniques have been used for the control of nuclear spins in molecules [7, 138, 139, 6, 140], which proved very successful for realizing QC with such nuclear spin qubits [6, 7] (see also Table A3). A wellknown example is the factorization of $N=15$ using Shor's algorithm [141]. Nuclear spin qubits have long coherence times ( $>1 \mathrm{~s}$ ) and high-fidelity quantum gates have been demonstrated [6]. The coherent control of up to 12 qubits has also been realized [140]. However, this approach to QC proved difficult to scale up to tens or hundreds of qubits, so NMR techniques are now being applied for the control of nuclear spins in semiconductors. One direction is solid-state NMR [138], but NMR is also merging with Electron Spin Resonance (ESR) methods, so it also becomes relevant for NV centers in diamond and for phosphorus in silicon QC.

\section{Comparing natural and artificial atoms}

The main characteristics of natural and artificial atoms are displayed in Tables 1 and 2 . In Table 1: $T_{1}$ (relaxation time) is the average time that the system takes for its excited state to decay to the ground state; $T_{2}$ (decoherence or dephasing time) represents the average time over which the qubit energy-level difference does not vary. We denote by $Q_{1}$ (quality factor) the number of one-qubit quantum gates that can be realized within the time $T_{2}$, and by $Q_{2}$ (quality factor) the number of two-qubit quantum gates that can be realized within the time $T_{2}$. For implementing $\mathrm{QC}$ we are mainly interested in the following aspects: controllability, scalability and interface-ability. The latter will also be discussed in the following section.

\section{Box 1: Natural and artificial atoms}

Both natural and artificial atoms exhibit discrete energy levels, which are modified in the presence of external fields $(E \neq 0)$. The applied external fields drive coherent quantum oscillations between the specific energy levels which can be used to encode the qubit states. Artificial atoms can be engineered to have certain transition frequencies while in natural atoms these are fixed.

The qubit energy-level splittings are comparable for natural and artificial atoms - mi- 


\begin{tabular}{|l|l|l|l|}
\hline & Natural atoms & \multicolumn{2}{c|}{ Artificial atoms } \\
\hline$E=0$ & Atoms and ions & Quantum dots & \\
\hline $\mathrm{E} \neq 0$ & & & \\
\hline
\end{tabular}

crowave frequencies (for ions and superconducting circuits) and optical frequencies (for neutral atoms, ions and some semiconductor quantum dots). Box 1 displays schematically the potential energies and discrete energy levels for natural and artificial atoms in the absence $(E=0)$ and in the presence $(E \neq 0)$ of an external field. While natural atoms are usually driven using optical or microwave radiation, artificial atoms like superconducting circuits can be driven by currents and voltages, magnetic fields, as well as microwave photons. Optically-driven artificial atoms, such as some semiconducting quantum dots, have also been demonstrated. Artificial atoms can be engineered to have a large dipole moment or particular transition frequencies. Depending on the intended application this tunability may prove quite useful.

In natural atoms, motional states can also be exploited for encoding the qubits or as data bus. The motional frequency can be controlled, but the cooling of these modes is usually necessary if they are to be used for QC purposes. For artificial atoms, resonators can play a similar role to the motional modes. The frequency of these resonators can also be controlled, and they can be cooled much like atoms. For instance, the temperature of superconducting circuits can be decreased using cooling techniques inspired from atomic physics, such as sideband or Sisyphus cooling [142, 143]. Natural atoms have many energy levels which can be used to encode information. Levels that are well-protected against decoherence (i.e., magnetic-field-independent hyperfine transitions [144]) could be used for memory qubits, while fast transitions could be used for implementing twoqubit gates. Furthermore, realizing qudits in natural atoms is straightforward.

Unlike natural atoms of the same species, which are indistinguishable, no two artificial atoms will be perfectly alike. With the latest advances in microfabrication, 
artificial atoms can be made with increasing accuracy and uniformity. However, this is an extra challenge. While natural atoms are readily available and one only needs to trap them by means of optical or electrical fields and then cool them down to low temperatures, artificial atoms have to be carefully designed and fabricated. Furthermore, atom and ion trapping technologies have been in use for quite a while, but for artificial atoms the techniques are more recent.

Artificial atoms can be produced in large numbers and "wired" together on a chip. Therefore, extending current experiments to large numbers of artificial atoms should, in principle, not be a problem. Neutral atoms can be loaded by thousands or millions in optical lattices; however, individual addressing has not yet been fully demonstrated [29]. Meanwhile, in the case of ions, although several proposals are available, scaling to large numbers is a challenge. Natural atoms are not wired so they can form almost any $2 \mathrm{D}$ or $3 \mathrm{D}$ configuration; however, for artificial atoms the wiring itself may impose some geometric limitations. Neutral atoms and trapped ions qubits can also be moved around easily. This flexibility may prove advantageous for certain applications.

Both natural and artificial atoms can be coupled with photons via cavities QED [4, 3, 86], which could provide a means of realizing large scale QC and long distance quantum communication (see also [145]). The physics of cavity QED is the same regardless of the nature of the atom or cavity, but, for artificial atoms (e.g., circuit QED) the coupling strength is several orders of magnitude larger than for natural atoms [4, 3, 86]. Several exciting experiments demonstrating the coupling between cavities and natural or artificial atoms have been performed (see, for instance, [146, 147, 148, 83, 80] and the review in [102]).

As for the operating conditions, natural atoms can be coherently manipulated only in ultrahigh-vacuum at very low temperatures $(\mathrm{nK}-\mu \mathrm{K}$ for neutral atoms and $\mathrm{mK}$ for ions). Artificial atoms are also operated at low temperatures ( $\mathrm{mK}$ in the case of superconducting circuits or a few $\mathrm{K}$ for semiconductor quantum dots), but there are some candidates for room-temperature qubits, including very long coherence times for NV centers in diamond (note that their $T_{1}$ is temperature dependent). 

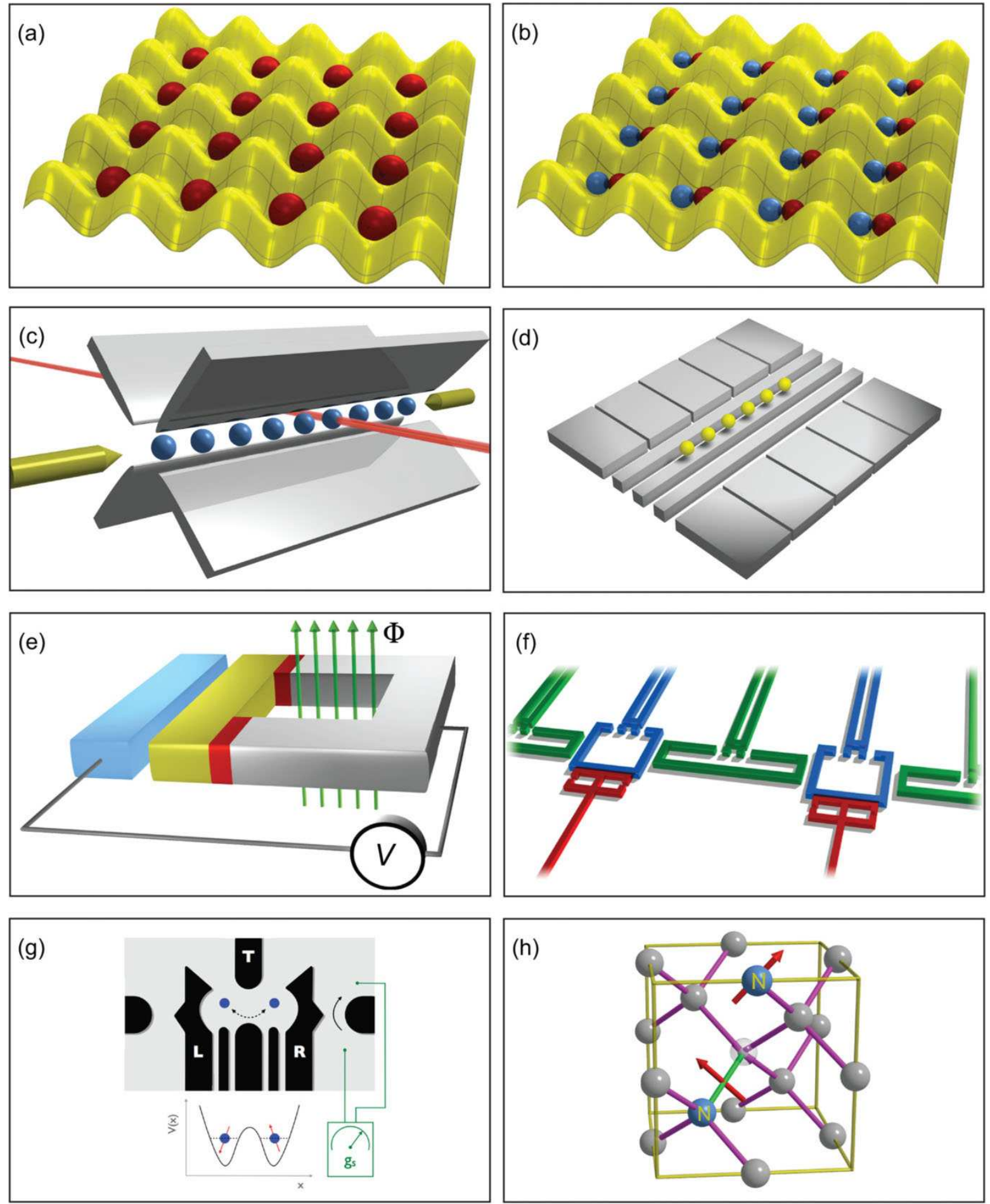

(h)

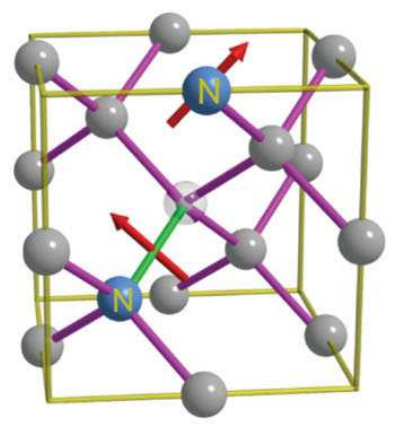




\section{Box 2: Quantum bits}

Quantum bits can be constructed using a variety of different possible building blocks, of various sizes and properties. As a result, each technology has its unique advantages and challenges.

(a,b) Hundreds of thousands of neutral atoms can be trapped and cooled at the minima of an optical lattice - the periodic potential created by interfering counterpropagating laser beams. The long-lived internal energy levels of neutral atoms are used to encode quantum information. Neutral atom qubits can be manipulated with laser radiation and observed via their laser-induced fluorescence. The typical separation between lattice sites is $<1 \mu \mathrm{m}$, which makes individual addressing challenging. Neutral atoms interact weakly with the environment, which protects them from decoherence. There are several mechanisms for entangling neutral atoms: through state-dependent displacement of the lattice, that results in a highly entangled many-qubit state created in a single operation; through exchange interactions; or via the interaction between two atoms in a double-well potential. Neutral atoms in optical lattices are ideal systems for quantum simulation. (a) illustrates the idea of trapping neutral atoms in periodic optical potentials; one neutral atom - qubit is trapped at each lattice site; (b) shows one possible mechanism for creating multi-particle entanglement starting with two atoms in different spin states, trapped in each lattice site.

$(\mathbf{c}, \mathbf{d})$ Ions trapped in electro-magnetic fields have been used to encode and manipulate quantum information. The internal energy levels representing the qubit basis states are long-lived and can be easily excited with laser radiation. The typical distance between trapped ions is $5 \mu \mathrm{m}$ or more which facilitates addressing and readout of individual ions. High-efficiency readout is achieved by monitoring the laser-induced fluorescence. Ions in the same potential have a common center-of-mass vibrational mode that can be used as data bus to realize entangling operations. Many-particle entanglement and high-fidelity two-qubit gates have already been demonstrated in experiment. Panel (c) shows a linear trap, while (d) a planar trap. These recently developed micrometer-scale ion traps (d) provide flexibility in manipulating the positions of the ions in two and three dimensions. Nowadays the main focus is on scaling these experiments to large numbers of ions. This can be achieved by moving the ions in the trapping potentials around in complex microstructures, trapping single ions at specific locations in custom-designed lattice geometries created in arrays of microtraps, or by entangling the ions with flying qubits (photons). 


\begin{abstract}
Box 2: Quantum bits (Continued)
(e,f) Superconducting qubits are micrometer-sized electric circuits based on Josephson junctions. A superconducting qubit (e) can be manipulated using the applied electric voltage $V$ and magnetic flux $\Phi$. Similarly, the qubit can be read out through the small electric or magnetic signal that it produces. Additional circuit elements, called couplers, can be used to provide tunable interactions between the qubits, as shown in (f), allowing the creation of entanglement and the performance of two-qubit gates. Decoherence times have improved from the nanosecond to the microsecond scale over the past decade and are expected to improve further in the future.

$(\mathbf{g}, \mathbf{h})$ Spins in solids arise in a number of distinct realizations. The collective spin state of two electrons trapped in a sub-micrometer-scale semiconductor-based double quantum dot structure can be used as a qubit, as shown in (g). In the traditional approach, magnetic fields are used to manipulate the qubit, but recent techniques using electric fields and exploiting the exchange and spin-orbit interactions have been developed as well. The qubit is readout by monitoring its response to an applied electric signal. Nitrogen-vacancy (NV) centers in diamond, shown in (h), also provide alternative spin qubits. The spin of one electron in the NV chemical bond can be manipulated and read out using magnetic fields and optical-frequency electromagnetic fields. These qubits have long coherence times, on the millisecond timescale. It would be highly desirable to controllably place multiple qubits in an ordered arrangement in the diamond crystal and couple them to each other, such that entanglement and two-qubit gates would be achieved.
\end{abstract}

\title{
7. Photons
}

Photons can also make good qubits and they can carry quantum information over long distances hardly being affected by noise or decoherence. The qubit states can be encoded, for example, in the polarization of a single photon, and one-qubit gates can be easily realized with optical elements [11, 149]. Unfortunately optical QC has a serious drawback: the difficulty in implementing two-qubit gates. Realizing the nonlinearity required for entangling two qubits is challenging, so alternatives such as the teleportation of nondeterministic quantum gates have been investigated [149]. While this approach is still impractical due to the large amount of required resources, another solution may be found in measurement-based QC.

For the moment photons may not be practical as memory or computation qubits, but they are certainly the best "flying qubits". Recent advances in quantum communication and, in particular, quantum key distribution are reviewed in [10]. 


\section{Hybrids}

Exploiting the advantages of both natural and artificial atoms in hybrid systems provides exciting prospects for realizing QC. For instance, ions [150, 151] and atoms [152, 153] interfaced with superconducting circuits are now being investigated. As recent results point out neutral atoms and ions could also be interfaced with each other [154, 155]. While cavity QED with atoms and ions has been studied for some time now [145, 86], solid-state cavity QED is more recent [83, 80, 148, 86]. For natural atoms strong coupling has been demonstrated [146, 147]. As mentioned before, in circuit QED the coupling strength is many orders of magnitude larger than in cavity QED, which is very promising for the study of quantum optics on a chip. As shown in Table 3, all systems discussed in the previous sections can be coupled with other systems. It is interesting to note that superconducting circuits can be coupled with both different types of natural atoms, spins in solids [156, 157, 158] and with photons.

Natural atoms, with their long decoherence times, are envisaged by many as quantum memories [159], while the tunable artificial atoms may be used for the "quantum processing unit". Both natural and artificial atoms may be coupled with photons via a cavity. Note that a necessary requirement is for the coupling timescale to be shorter than the decoherence time. Such cavities could be used as input/output interfaces and for long distance communication. Perhaps the first functional quantum computer will be a complex hybrid system made of natural atoms, artificial atoms, and photons. Such a hybrid device is represented schematically in Figure 1. Several types of hybrids are discussed in [160].

\section{Prospects}

In both natural and artificial atoms, almost all the basic requirements for realizing QC 161 have been demonstrated (i.e., (i) a scalable system with well-characterized qubits; (ii) initialization of the qubits; (iii) reasonably long decoherence times; (iv) a universal set of quantum gates; (v) measurement of the qubits). Tables 1-6 and Figure 2 provide a brief snapshot of the progress and current experimental status for several types of qubits.

The current challenges are to attain increased controllability (and minimize decoherence) and scale the existing systems to tens and hundreds of qubits and many-gate operations. At this stage, new milestones, such as the creation of manyparticle entangled states, the implementation of small quantum algorithms, and other applications (e.g., quantum simulation), and the realization of quantum communication by interfacing the qubits with photons, are being targeted.

"Quantum supercomputers" for factorizing large numbers are still a distant goal. The first-generation of practical quantum computers may be either specialized devices for scientific applications like quantum simulations [162], or integrated in complex quantum networks [145]. As the very positive results summarized above point out, the 


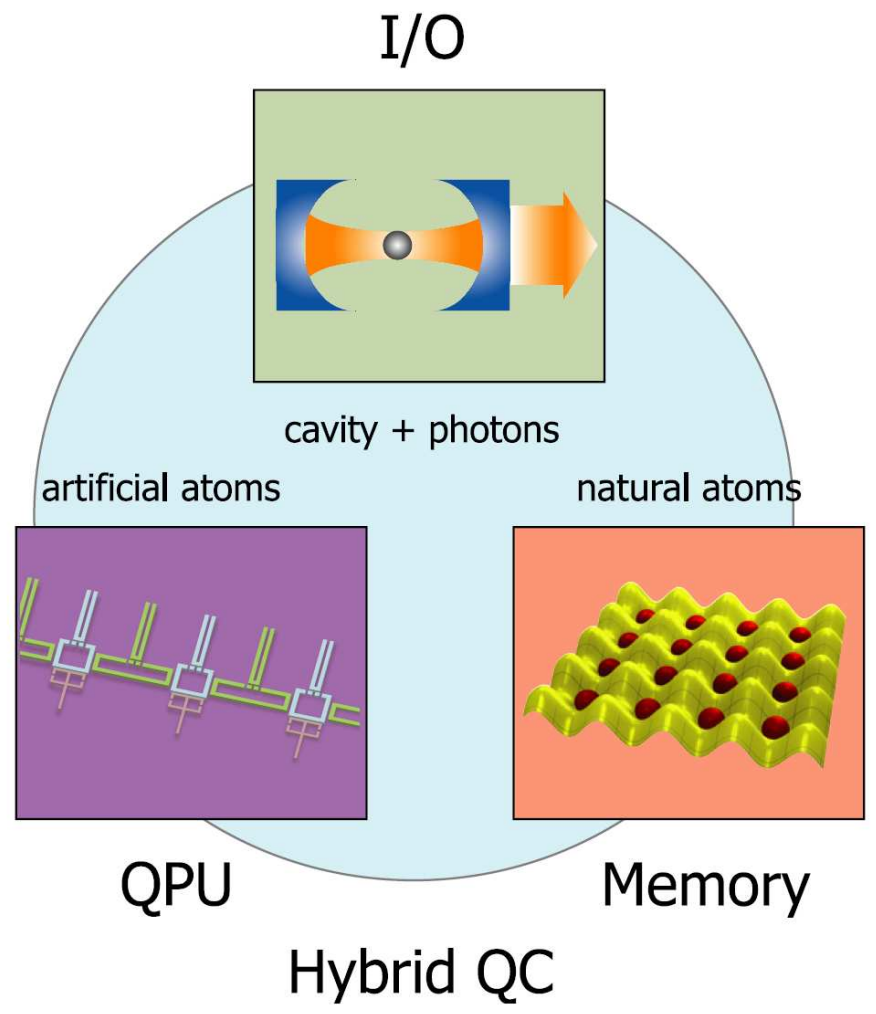

Figure 1. Schematic representation of a hybrid device consisting of natural atoms as quantum memory, artificial atoms as "quantum processing unit" (QPU), and an input/output (I/O) photonic interface.

first-generation quantum computers may be available in the near future. Furthermore, they may come as hybrids consisting of natural atoms, artificial atoms, and photons.

\section{Acknowledgments}

We thank R. Blatt, P. Grangier, L. Kouwenhoven, C. Marcus, A. Morello, W. Oliver, T. Porto, M. Saffman, D. Wineland and A. Yacoby for useful comments on the manuscript. FN acknowledges partial support from the Laboratory of Physical Sciences (LPS), National Security Agency (NSA), Army Research Office (ARO), Defense Advanced Research Projects Agency (DARPA), Air Force Office of Scientific Research (AFOSR), National Science Foundation (NSF) grant No.0726909, JSPS-RFBR contract No.0902-92114, Grant-in-Aid for Scientific Research (S), MEXT Kakenhi on Quantum Cybernetics, and the Funding Program for Innovative R\&D on Science and Technology (FIRST). 


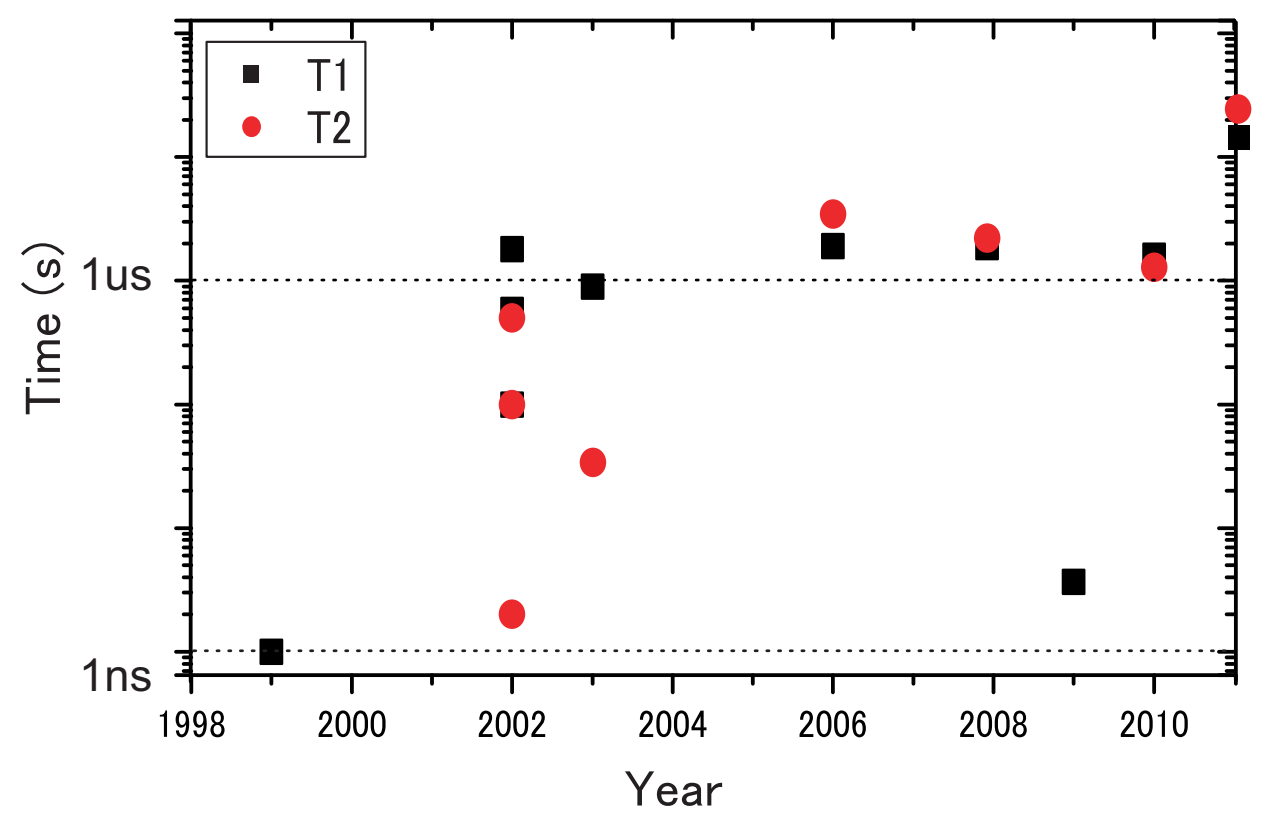

Figure 2. An example of the progress that has been achieved for superconducting circuits in the last decade. The decoherence time kept increasing, and the current trend promises decoherence times of the order of ms in the next couple of years. Visibility also increased and now it is larger than $95 \%$. The black squares show $T_{1}$ and the red $\operatorname{dots} T_{2}$. 
Table 1. Comparison between natural and artificial atoms.Note: (a) distance between qubits for NV centers and ${ }^{(b)}$ typical distances between quantum dots.

\begin{tabular}{|c|c|c|c|c|}
\hline & \multicolumn{2}{|c|}{ Natural atoms } & \multicolumn{2}{|c|}{ Artificial atoms } \\
\hline & Neutral atoms & Trapped ions & Supercond. circuits & Spins in solids \\
\hline Energy gap & $\begin{array}{l}\mathrm{GHz} \text { (hyperfine), } \\
10^{14} \mathrm{~Hz} \text { (optical) }\end{array}$ & $\begin{array}{l}\mathrm{GHz} \text { (hyperfine), } \\
10^{14} \mathrm{~Hz} \text { (optical) }\end{array}$ & $1-10 \mathrm{GHz}$ & $\begin{array}{c}\mathrm{GHz} \\
10^{13} \mathrm{~Hz}\end{array}$ \\
\hline Photon & Optical, MW & Optical, MW & MW & $\begin{array}{l}\text { Optical, MW, } \\
\text { infrared }\end{array}$ \\
\hline Dimension & $\sim 2 \AA$ & $\sim 2 \AA$ & $\sim \mu \mathrm{m}$ & $\sim \mathrm{nm}$ \\
\hline $\begin{array}{l}\text { Distance } \\
\text { between qubits }\end{array}$ & $<1 \mu \mathrm{m}$ & $\sim 5 \mu \mathrm{m}$ & $\sim \mu \mathrm{m}$ & $\sim 10 \mathrm{~nm}^{(\mathrm{a})}, \sim 100 \mathrm{~nm}{ }^{(\mathrm{b})}$ \\
\hline $\begin{array}{l}\text { Operating } \\
\text { temperature }\end{array}$ & $\mathrm{nK}-\mu \mathrm{K}$ & $\mu \mathrm{K}-\mathrm{mK}$ & $\sim \mathrm{mK}$ & $\mathrm{mK}-300 \mathrm{~K}$ \\
\hline $\begin{array}{l}\text { Qubit } \\
\text { interactions }\end{array}$ & $\begin{array}{l}\text { Collisions, } \\
\text { exchange }\end{array}$ & Coulomb & $\begin{array}{l}\text { Capacitive, } \\
\text { inductive }\end{array}$ & $\begin{array}{c}\text { Coulomb, } \\
\text { exchange, } \\
\text { dipolar }\end{array}$ \\
\hline Cooling & $\begin{array}{c}\text { Doppler, } \\
\text { Sisyphus, } \\
\text { evaporative }\end{array}$ & $\begin{array}{l}\text { Doppler, } \\
\text { sideband }\end{array}$ & Cryogenic & Cryogenic \\
\hline Cavity & $\begin{array}{l}\text { Optical, } \\
\text { MW }\end{array}$ & $\begin{array}{l}\text { Optical, } \\
\text { vib. modes }\end{array}$ & $\begin{array}{c}\text { Transmission } \\
\text { line, LC } \\
\text { circuit }\end{array}$ & $\begin{array}{l}\text { Optical, } \\
\text { MW }\end{array}$ \\
\hline
\end{tabular}


Table 2. Comparison between natural and artificial atoms in the view of implementing quantum computation. Hereafter, MW stands for microwaves and SC for superconducting. (a) large entangled states can also be realized with collisional gates; (b) entanglement of the ground state of four qubits; (c) NV centers in diamond; (d) $T_{1}$ for the vibrational modes; (e) $T_{1}$ for the internal hyperfine states; (f) of the order of $\mathrm{ms}$ for $\mathrm{NV}$ centers at room temperature and of the order of minutes at $1 \mathrm{~K}$; of the order of seconds for P:Si; ${ }^{g}$ ) in optical clocks $T_{1}, T_{2}>10$ minutes has been observed;

(i) only generated for one and two resonators and not for many qubits.

\begin{tabular}{|c|c|c|c|c|}
\hline & \multicolumn{2}{|c|}{ Natural atoms } & \multicolumn{2}{|c|}{ Artificial atoms } \\
\hline & Neutral atoms & Trapped ions & Supercond. circuits & Spins in solids \\
\hline \# entangled qubits & $2^{(a)}$ & 14 & $3\left(4^{(b)}\right)$ & $1\left(3^{(\mathrm{c})}\right)$ \\
\hline One-qubit gates fidelity & $99 \%$ & $99 \%$ & $99 \%$ & $>73 \%\left(>99 \%{ }^{(\mathrm{c})}\right)$ \\
\hline Two-qubit gates fidelity & $>64 \%$ & $99.3 \%$ & $>90 \%$ & $90 \%(\mathrm{c})$ \\
\hline Entangled states & Bell & $\begin{array}{l}\text { Bell, GHZ, } \\
\text { W, cat }\end{array}$ & $\begin{array}{l}\text { Bell, GHZ }{ }^{(i)} \\
\text { W, cat }\end{array}$ & $\mathrm{GHZ}^{(\mathrm{c})}$ \\
\hline Measurement efficiency & $99.9 \%$ & $99.9 \%$ & $>95 \%$ & $99 \%$ \\
\hline$T_{1}$ & $\sim \mathrm{s}$ & $\begin{array}{l}\sim 100 \mathrm{~ms}{ }^{(\mathrm{d})} \\
>20 \mathrm{~ms}\end{array}$ & $10 \mu \mathrm{s}$ & $\sim 1 \mathrm{~s}^{(\mathrm{f})}$ \\
\hline$T_{2}$ & $\sim 40 \mathrm{~ms}$ & $1000 \mathrm{~s}(\mathrm{~g})$ & $20 \mu \mathrm{s}$ & $200 \mu \mathrm{s}(\mathrm{f})$ \\
\hline$Q_{1}$ & $\sim 10^{4}$ & $\sim 10^{13}$ & $\sim 10^{5}$ & $\begin{array}{c}\sim 10^{3}-10^{4} \\
\left(10^{6}(\mathrm{c})\right)\end{array}$ \\
\hline$Q_{2}$ & $\sim 4 \times 10^{4}$ & $\begin{array}{c}2 \times 10^{2}-2 \times 10^{3} \\
\sim 2 \times 10^{4}\end{array}$ & $>100$ & tbd \\
\hline Interfaceable with & $\begin{array}{l}\text { photons, SC } \\
\text { circuits }\end{array}$ & $\begin{array}{l}\text { photons, SC } \\
\text { circuits }\end{array}$ & $\begin{array}{c}\text { photons, atoms, } \\
\text { ions }\end{array}$ & photons \\
\hline
\end{tabular}


Table 3. Interfacing different types of qubits for future scalability or realizing long-range quantum communication. The asterisk denotes the cases that have been experimentally realized and the dash means that, to the best of our knowledge, no proposal exists yet.

\begin{tabular}{lccccc}
\hline & Atoms & Ions & Cavity & Spins & SC \\
\hline Atoms & & $\checkmark$ & $\checkmark *$ & - & $\checkmark *$ \\
Ions & $\checkmark$ & & $\checkmark *$ & - & $\checkmark$ \\
Cavity & $\checkmark *$ & $\checkmark *$ & & $\checkmark$ & $\checkmark *$ \\
Spins & - & - & $\checkmark$ & & $\checkmark$ \\
SC & $\checkmark *$ & $\checkmark$ & $\checkmark *$ & $\checkmark$ & \\
\hline
\end{tabular}

Table 4. Coherence times of superconducting qubits.

\begin{tabular}{ccccc}
\hline Year & T1 & T2 (echo) & Qubit & Ref. \\
\hline $\mathbf{1 9 9 9}$ & $1 \mathrm{~ns}$ & - & Charge & {$[65]$} \\
$\mathbf{2 0 0 2}$ & $580 \mathrm{~ns}$ & $2 \mathrm{~ns}$ & Charge & $66]$ \\
$\mathbf{2 0 0 2}$ & $100 \mathrm{~ns}$ & $100 \mathrm{~ns}$ & Phase & {$[67]$} \\
$\mathbf{2 0 0 2}$ & $1.8 \mu \mathrm{s}$ & $500 \mathrm{~ns}$ & Hybrid (charge/phase) & {$[68$} \\
$\mathbf{2 0 0 3}$ & $0.9 \mu \mathrm{s}$ & $30 \mathrm{~ns}$ & Flux & $69]$ \\
$\mathbf{2 0 0 6}$ & $1.9 \mu \mathrm{s}$ & $3.5 \mu \mathrm{s}$ & Flux & {$[77$} \\
$\mathbf{2 0 0 8}$ & $1.87 \mu \mathrm{s}$ & $2.22 \mu \mathrm{s}$ & Hybrid (charge/phase) & {$[85$} \\
$\mathbf{2 0 0 9}$ & $350 \mathrm{~ns}$ & - & Flux & {$[89$} \\
$\mathbf{2 0 1 0}$ & $1.6 \mu \mathrm{s}$ & $1.3 \mu \mathrm{s}$ & Hybrid (phase/flux) & {$[96$} \\
$\mathbf{2 0 1 1}$ & $12 \mu \mathrm{s}$ & $23 \mu \mathrm{s}$ & Flux & {$[98$} \\
$\mathbf{2 0 1 1}$ & $0.2 \mathrm{~ms}$ & - & Charge & {$[163$} \\
\hline
\end{tabular}


Table 5. Progress in the implementation of superconducting qubits quantum gates.

\begin{tabular}{|c|c|c|c|c|}
\hline Year & Operation & Qubits & Mechanism & Ref. \\
\hline 2003 & CNOT gate & 2 & $\begin{array}{l}\text { Direct coupling; } \\
\text { gate relies on zz component }\end{array}$ & 71 \\
\hline 2003 & Entangled energy levels & 2 & Direct xy coupling & 70 \\
\hline 2005 & iSWAP; Entanglement & 2 & Direct xy coupling & 73 \\
\hline 2006 & iSWAP; Entanglement & 2 & Direct xy coupling & 76 \\
\hline 2006 & Entangled energy levels & 4 & Direct coupling & 75 \\
\hline 2006-7 & Controllable coupling & 2 & $\begin{array}{l}\text { Coupling mediated by } \\
\text { additional circuit element }\end{array}$ & [74, 78 \\
\hline 2007 & CNOT gate & 2 & $\begin{array}{l}\text { Direct coupling; } \\
\text { gate relies on zz component }\end{array}$ & 82 \\
\hline 2007 & iSWAP & 2 & $\begin{array}{l}\text { xy coupling to cavity; } \\
\text { gate mediated by cavity }\end{array}$ & 83 \\
\hline 2007 & iSWAP & 2 & xy coupling mediated by cavity & 80 \\
\hline 2007 & iSWAP & 2 & $\begin{array}{l}\text { Coupling mediated by additional } \\
\text { circuit element; gate relies on xy coupling }\end{array}$ & 81 \\
\hline 2009 & CPhase & 2 & $\begin{array}{l}\text { zz coupling mediated by } \\
\text { auxilliary energy levels }\end{array}$ & 88 \\
\hline 2010 & Entanglement & 3 & xy coupling & 90 \\
\hline 2010 & Entanglement & 3 & $\begin{array}{l}\text { zz coupling mediated by } \\
\text { auxilliary energy levels }\end{array}$ & 91 \\
\hline 2011 & 3-qubit gate & 3 & $\begin{array}{l}\text { Coupling mediated by } \\
\text { auxilliary energy levels }\end{array}$ & 97 \\
\hline
\end{tabular}


Table 6. Progress in the number of qubits and fidelities for different operations on trapped ions. CZ stands for the Cirac-Zoller scheme [164], and MS for the MølmerSørensen scheme [165].

\begin{tabular}{lccccc}
\hline Year & Operation & Mechanism & Qubits & Fidelity & Ref. \\
\hline $\mathbf{1 9 9 8}$ & Entanglement & $\mathrm{CZ}$ & 2 & $70 \%$ & {$[40]$} \\
$\mathbf{2 0 0 0}$ & Entanglement & $\mathrm{MS}$ & 2 & $83 \%$ & {$[42]$} \\
& & & 4 & $57 \%$ & \\
$\mathbf{2 0 0 3}$ & CNOT gate & $\mathrm{CZ}$ & 2 & $71.3 \%$ & {$[43]$} \\
$\mathbf{2 0 0 3}$ & Entanglement & Geometric & 2 & $97 \%$ & {$[45]$} \\
$\mathbf{2 0 0 5}$ & Entanglement & $\mathrm{CZ}$ & 4 & $>76 \%$ & {$[52]$} \\
& & & 5 & $>60 \%$ & \\
$\mathbf{2 0 0 5}$ & Entanglement & $\mathrm{CZ}$ & 4 & $85 \%$ & {$[51]$} \\
& & & 5 & $76 \%$ & \\
& & & 6 & $79 \%$ & \\
& & & 7 & $76 \%$ & \\
$\mathbf{2 0 0 6}$ & CNOT gate & $\mathrm{CZ}$ & 2 & $92.6 \%$ & {$[53]$} \\
$\mathbf{2 0 0 8}$ & Entanglement & $\mathrm{MS}$ & 2 & $99.3 \%$ & {$[56]$} \\
$\mathbf{2 0 0 9}$ & Toffoli gate & $\mathrm{CZ}$ & 3 & $74 \%$ & {$[60]$} \\
$\mathbf{2 0 1 0}$ & Entanglement & $\mathrm{MS}$ & 10 & $62.9 \%$ & {$[64]$} \\
& & & 12 & $39.6 \%$ & \\
\hline & & & 14 & $46.3 \%$ & \\
\hline
\end{tabular}




\section{Appendix A. Tables summarizing the main characteristics of different systems in the view of realizing $\mathrm{QC}$}

In the following tables, $T_{1}$ (relaxation time) is defined as the average time that the system takes for its excited state to decay to the ground state; $T_{2}$ (decoherence time) represents the average time over which the qubit energy-level difference does not vary; $Q_{1}$ (quality factor) represents the number of one-qubit quantum gates that can be realized within the time $T_{2} ; Q_{2}$ (quality factor) represents the number of two-qubit quantum gates that can be realized within the time $T_{2}$. The following abbreviation is used: tbd for "to be demonstrated" 
Table A1. Neutral atoms.

\begin{tabular}{|c|c|}
\hline & Neutral atoms \\
\hline$\overline{\text { Qubits }}$ & $\begin{array}{l}\text { Internal states (ground hyperfine states); } \\
\text { Motional states (trapping potential eigenstates) }\end{array}$ \\
\hline Scalability & Demonstrated in optical lattices; possible in arrays of cavities, atom chips \\
\hline Initialization & Both internal (optical pumping) and motional (laser cooling) states \\
\hline Long coherence time & Several seconds $19,30,15$ \\
\hline Universal quantum gates & One-, two-qubit gates (several proposals) \\
\hline Measurement & Fluorescence: "quantum jump" technique \\
\hline \multicolumn{2}{|l|}{ Fabrication } \\
\hline Material & Trapped neutral atoms: Rb, Li, K, Cs, etc \\
\hline Well controlled fabrication & yes \\
\hline Flexible geometry & yes (especially in optical lattices) \\
\hline Distance between qubits & A few hundred $\mathrm{nm}$ to a few $\mu \mathrm{m}[1]$ \\
\hline \multicolumn{2}{|l|}{ Operation } \\
\hline Qubits demonstrated & $>10^{6}$ (stored), 2 (entangled) \\
\hline Superposition/Entangled states & yes/yes \\
\hline One-qubit gates (Fidelity) & yes $(99.98 \%)$ \\
\hline Two-qubit gates (Fidelity) & yes (SWAP >64\% [20]); CNOT (73\% [33]) \\
\hline Operation temperature & From $\mathrm{nK}$ to $\mu \mathrm{K}$ \\
\hline \multicolumn{2}{|l|}{$\overline{\text { Readout }}$} \\
\hline Readout (Fidelity) & Laser-induced fluorescence (99.9\%) \\
\hline Single-qubit readout possible & yes \\
\hline \multicolumn{2}{|l|}{ Manipulation } \\
\hline Controls & Optical fields, microwave \\
\hline Types of operations & One-, two-qubit gates, entanglement \\
\hline Individual addressing & To be demonstrated [24, 29, 35, 32, 31] \\
\hline \multicolumn{2}{|l|}{ Decoherence } \\
\hline Decoherence sources & Photon scattering, heating, stray fields, laser fluctuations \\
\hline$T_{1}$ & $\sim \mathrm{s}$ \\
\hline$T_{2}$ & $\sim 40 \mathrm{~ms}$ \\
\hline$Q_{1}$ & $\sim 10^{4}$ \\
\hline$Q_{2}$ & $\sim 40000$ \\
\hline
\end{tabular}


Table A2. Trapped ions.

\begin{tabular}{|c|c|}
\hline & Trapped ions \\
\hline Qubits & $\begin{array}{l}\text { Internal states (hyperfine or Zeeman sublevels, optical); } \\
\text { Motional states (collective oscillations) }\end{array}$ \\
\hline Scalability & Ion shuttling, arrays, photon interconnections, long strings \\
\hline Initialization & Both internal (optical pumping) and motional (laser cooling) states \\
\hline Long coherence time & Internal: hyperfine $>20 \mathrm{~s}$, optical $>1 \mathrm{~s} ;$ Motional: $\sim 100 \mathrm{~ms}$ \\
\hline Universal quantum gates & One-, two-, three-qubit gates \\
\hline Measurement & Fluorescence: "quantum jump" technique \\
\hline \multicolumn{2}{|l|}{ Fabrication } \\
\hline Material & Atomic ions: $\mathrm{Ca}^{+}, \mathrm{Be}^{+}, \mathrm{Ba}^{+}, \mathrm{Mg}^{+}$, etc \\
\hline Well controlled fabrication & yes \\
\hline Flexible geometry & yes \\
\hline Distance between qubits & A few $\mu \mathrm{m}$ to tens of $\mu \mathrm{m}$ \\
\hline \multicolumn{2}{|l|}{ Operation } \\
\hline Qubits demonstrated & $10-10^{3}$ (stored), 14 (entangled) 64 \\
\hline Superposition/Entangled states & yes/yes (2-14 ions, fidelities 99.3\%-46\%) 64 \\
\hline One-qubit gates (Fidelity) & yes $(99 \%)$ \\
\hline Two-qubit gates (Fidelity) & yes $(\mathrm{CNOT}>99.3 \%$ [56; Toffoli $71.3 \%$ [60; gate time $1.5 \mathrm{~ms})$ \\
\hline Operation temperature & From $\mu \mathrm{K}$ to $\mathrm{mK}$ \\
\hline \multicolumn{2}{|l|}{ Readout } \\
\hline Readout (Fidelity) & Laser-induced fluorescence (99.9\%) \\
\hline Single-qubit readout possible & yes \\
\hline \multicolumn{2}{|l|}{ Manipulation } \\
\hline Controls & Optical, microwave, electric/magnetic fields \\
\hline Types of operations & One-, two-, three-qubit gates, entanglement \\
\hline Individual addressing & yes \\
\hline \multicolumn{2}{|l|}{ Decoherence } \\
\hline Decoherence sources & Heating, spontaneous emission, laser, magnetic field fluctuations \\
\hline$T_{1}$ & a few minutes (hyperfine), $1 \mathrm{~s}$ (optical), $100 \mathrm{~ms}$ (motional) \\
\hline$T_{2}$ & $15 \mathrm{~s}$ \\
\hline$Q_{1}$ & $\sim 10^{13}$ (single-qubit gate $50 \mathrm{ps}$ ) 63 \\
\hline$Q_{2}$ & $\sim 20000($ MS gate $50 \mu \mathrm{s})[56$; $\sim 200(\mathrm{CZ}$ gate $500 \mu \mathrm{s})[53$ \\
\hline
\end{tabular}


Table A3. Nuclear spins manipulated by Nuclear Magnetic Resonance (NMR).

\begin{tabular}{|c|c|}
\hline & NMR \\
\hline Qubits & Nuclear spin \\
\hline Scalability & Not available in liquid-state NMR; possible for solid-state NMR \\
\hline Initialization & Demonstrated \\
\hline Long coherence time & $>1 \mathrm{~s}$ \\
\hline Universal quantum gates & One-, two-, three-qubit gates \\
\hline Measurement & Single-qubit measurement not available \\
\hline \multicolumn{2}{|l|}{ Fabrication } \\
\hline Material & Organic molecules (alanine, chloroform, cytosine) \\
\hline Well controlled fabrication & yes \\
\hline Flexible geometry & no \\
\hline Distance between qubits & $\sim \AA$ \\
\hline \multicolumn{2}{|l|}{ Operation } \\
\hline Qubits demonstrated & 7, 12 (entangled) liquid-state [140]; >100 (correlated) solid-state \\
\hline Superposition/Entangled states & yes/yes \\
\hline One-qubit gates (Fidelity) & yes $(>98 \%)$ \\
\hline Two-qubit gates (Fidelity) & yes $(>98 \%$ CNOT and SWAP) \\
\hline Operation temperature & Room temperature \\
\hline \multicolumn{2}{|l|}{ Readout } \\
\hline Readout (Fidelity) & Voltage in neighboring coil induced by precessing spins, $99.9 \%$ \\
\hline Single-qubit readout possible & no \\
\hline \multicolumn{2}{|l|}{ Manipulation } \\
\hline Controls & RF pulses \\
\hline Types of operations & One-, two-, three-qubit gates \\
\hline Individual addressing & no \\
\hline \multicolumn{2}{|l|}{ Decoherence } \\
\hline Decoherence sources & Coupling errors \\
\hline$T_{1}$ & $>1 \mathrm{~s}$ (liquid-state); > $1 \mathrm{~min}$ (solid-state) \\
\hline$T_{2}$ & $\sim 1 \mathrm{~s}$ (liquid-state); $>1 \mathrm{~s}$ (solid-state) \\
\hline \multicolumn{2}{|l|}{$Q_{1}$} \\
\hline$Q_{2}$ & 100 (gate time $10 \mathrm{~ms}$ ) \\
\hline
\end{tabular}


Table A4. Superconducting circuits.

\begin{tabular}{|c|c|}
\hline & Superconducting circuits \\
\hline Qubits & Flux, phase states, charge; also hybrids \\
\hline Scalability & High potential for scalability \\
\hline Initialization & Demonstrated for all types of qubits \\
\hline Long coherence time & $\sim 10 \mu \mathrm{s}$ \\
\hline Universal quantum gates & One-, two-qubit gates \\
\hline Measurement & Individual measurement possible \\
\hline \multicolumn{2}{|l|}{ Fabrication } \\
\hline Material & Josephson junctions $\left(\mathrm{Al}-\mathrm{Al}_{x} \mathrm{O}_{y}-\mathrm{Al}, \mathrm{Nb}-\mathrm{Al}_{x} \mathrm{O}_{y} \mathrm{Nb}\right)$ \\
\hline Well controlled fabrication & yes \\
\hline Flexible geometry & yes \\
\hline Distance between qubits & $\sim \mu \mathrm{m}$ \\
\hline \multicolumn{2}{|l|}{ Operation } \\
\hline Qubits demonstrated & 128 (fabricated) [93, 3 (entangled) \\
\hline Superposition/Entangled states & yes/yes \\
\hline One-qubit gates (Fidelity) & yes $(99 \%)$ \\
\hline Two-qubit gates (Fidelity) & yes $(>90 \%) 88$ \\
\hline Operation temperature & $\mathrm{mK}$ \\
\hline \multicolumn{2}{|l|}{ Readout } \\
\hline Readout (Fidelity) & SET, SQUID (> 95\%) 84, cavity frequency shift [72] \\
\hline Single-qubit readout possible & yes \\
\hline \multicolumn{2}{|l|}{ Manipulation } \\
\hline Controls & Microwave pulses, voltages, currents \\
\hline Types of operations & One-, two-, three-qubit gates, entanglement \\
\hline Individual addressing & yes \\
\hline \multicolumn{2}{|l|}{ Decoherence } \\
\hline Decoherence sources & Electric and magnetic noise, $1 /$ f noise \\
\hline$T_{1}$ & $0.2 \mathrm{~ms}[163$ \\
\hline$T_{2}$ & $23 \mu \mathrm{s} 98$ \\
\hline$Q_{1}$ & $\sim 10^{5}$ \\
\hline$Q_{2}$ & $>100$ (gate time 10-50 ns) 88 \\
\hline
\end{tabular}


Table A5. Spins in solids. Here, QDs stand for quantum dots, NV centers for nitrogen-vacancy centers in diamond and P:Si for phosphorous on silicon. The asterisk * refers to room temperature.

\begin{tabular}{|c|c|}
\hline & Spins in solids \\
\hline Qubits & Electron spin; Electron and nuclear spins in NV centers in diamond, P:Si \\
\hline Scalability & High potential for scalability \\
\hline Initialization & Demonstrated \\
\hline Long coherence time & $>1 \mathrm{~s}(\mathrm{QDs}) ; \sim \mathrm{s}(\mathrm{NV}$ centers $), \sim 100 \mathrm{~s}(\mathrm{P}: \mathrm{Si})$ \\
\hline Universal quantum gates & One-qubit gates \\
\hline Measurement & Electrical, optical \\
\hline \multicolumn{2}{|l|}{ Fabrication } \\
\hline Material & GaAs, InGaAs (QDs), NV centers in diamond, P:Si \\
\hline Well controlled fabrication & yes \\
\hline Flexible geometry & yes \\
\hline Distance between qubits & $100-300 \mathrm{~nm}(\mathrm{QDs}) ; \sim 10 \mathrm{~nm}(\mathrm{NV}$ centers $)$ \\
\hline \multicolumn{2}{|l|}{ Operation } \\
\hline Qubits demonstrated & 1 (QDs), 3 (NV centers) 123 \\
\hline Superposition & yes \\
\hline One-qubit gates (Fidelity) & yes $(>73 \%$ QDs [112; $>>99 \%$ NV centers [130]) \\
\hline Two-qubit gates (Fidelity) & yes $(90 \%$ NV centers [108]) \\
\hline Operation temperature & From $\mathrm{mK}$ to a few $\mathrm{K}$ (QDs); room temperature (NV centers) \\
\hline \multicolumn{2}{|l|}{ Readout } \\
\hline Readout (Fidelity) & electrical, optical (90-92\%) \\
\hline Single-qubit readout possible & yes \\
\hline \multicolumn{2}{|l|}{ Manipulation } \\
\hline Controls & RF, optical pulses, electrical \\
\hline Types of operations & One-qubit gates (>73\% gate time $25 \mathrm{~ns})$ \\
\hline Individual addressing & yes \\
\hline \multicolumn{2}{|l|}{ Decoherence } \\
\hline Decoherence sources & Co-tunneling, charge noise, coupling with nuclear spins \\
\hline$T_{1}$ & $>1 \mathrm{~s}(\mathrm{QDs})[119 ;>5 \mathrm{~ms} *(\mathrm{NV}$ centers $)[123 ; ; 6 \mathrm{~s}$ [133] (P:Si); $100 \mathrm{~s}$ [134] (P:Si) \\
\hline$T_{2}$ & $\sim 270 \mu \mathrm{s}$ [129, 128; $\sim 1.8 \mathrm{~ms}(\mathrm{NV}$ centers) [124]; $\sim 60 \mathrm{~ms}$ [106] $(\mathrm{P}: \mathrm{Si}) ; 2 \mathrm{~s}[9](\mathrm{P}: \mathrm{Si})$ \\
\hline$Q_{1}$ & $\sim 10^{3}$ (gate time $\left.180 \mathrm{ps}\right) ; \sim 10^{4}$ (gate time $\left.30 \mathrm{ps}\right)[120] ;>10^{6}$ (gate time $\sim 1 \mathrm{~ns}$ ) \\
\hline$Q_{2}$ & tbd \\
\hline
\end{tabular}




\section{References}

Due to space limitations we list a small subset of recent, relevant papers, mostly experimental results. The very few theory papers cited here introduce parameters used in the experimental papers cited, and also in the tables (e.g., as in Table 6). For more references on the theoretical aspects, please refer to the various more specialized reviews listed below.

[1] I. Bloch. Quantum coherence and entanglement with ultracold atoms in optical lattices. Nature, 453:1016-1022, 2008.

[2] R. Blatt and D. J. Wineland. Entangled states of trapped atomic ions. Nature, 453:1008-1015, 2008.

[3] J. Clarke and F. K. Wilhelm. Superconducting quantum bits. Nature, 453:1031-1042, 2008.

[4] J. Q. You and F. Nori. Superconducting circuits and quantum information. Physics Today, $58(11): 42-47,2005$.

[5] R. Hanson and D. D. Awschalom. Coherent manipulation of single spins in semiconductors. Nature, 453:1043-1049, 2008.

[6] L. M. K. Vandersypen and I. L. Chuang. NMR techniques for quantum control and computation. Rev. Mod. Phys., 76:1037-1069, 2005.

[7] J. Baugh, J. Chamilliard, C. M. Chandrashekar, M. Ditty, A. Hubbard, R. Laflamme, M. Laforest, D. Maslov, O. Moussa, C. Negrevergne, M. Silva, S. Simmons, C. A. Ryan, D. G. Cory, J. S. Hodges, and C. Ramanathan. Quantum information processing using nuclear and electron magnetic resonance: review and prospects. arXiv:0710.1447v1, 2007.

[8] B. E. Kane. A silicon-based nuclear spin quantum computer. Nature, 393:133-137, 1998.

[9] J. J. L. Morton, A. M. Tyryshkin, R. M. Brown, S. Shankar, B. W. Lovett, A. Ardavan, T. Schenkel, E. E. Haller, J. W. Ager, and S. A. Lyon. Solid-state quantum memory using the 31P nuclear spin. Nature, 455:1085-1088, 2008.

[10] N. Gisin and R. Thew. Quantum communication. Nature Photonics, 1:165-171, 2007.

[11] P. Kok, W. J. Munro, K. Nemoto, T. C. Ralph, J. P. Dowling, and G. J. Milburn. Linear optical quantum computing with photonic qubits. Reviews of Modern Physics, 79:135, 2007.

[12] T. D. Ladd, F. Jelezko, Y. Nakamura R. Laflamme and, C. Monroe, and J. L. O'Brien. Quantum computers. Nature, 464:45-53, 2010.

[13] O. Mandel, M. Greiner, A. Widera, T. Rom, T. W. Hänsch, and I. Bloch. Controlled collisions for multi-particle entanglement of optically trapped atoms. Nature, 425:937-940, 2003.

[14] D. Schrader, I. Dotsenko, M. Khudaverdyan, Y. Miroshnychenko, A. Rauschenbeutel, and D. Meschede. Neutral atom quantum register. Phys. Rev. Lett., 93:150501, 2004.

[15] P. Treutlein, T. W. Hänsch P. Hommelhoff, T. Steinmetz, and J. Reichel. Coherence in microchip traps. Phys. Rev. Lett., 92:203005, 2004.

[16] D. Jaksch and P. Zoller. The cold atom Hubbard toolbox. Annals of Physics, 315:52-79, 2005.

[17] A. Micheli, G. K. Brennen, and P. Zoller. A toolbox for lattice-spin models with polar molecules. Nature Physics, 2:341-347, 2006.

[18] Y. Miroshnychenko, W. Alt, I. Dotsenko, L. Forster, M. Khudaverdyan, D. Meschede, D. Schrader, and A. Rauschenbeutel. Quantum engineering: An atom-sorting machine. Nature, 442:151, 2006.

[19] D. D. Yavuz, P. B. Kulatunga, E. Urban, T. A. Johnson, N. Proite, T. Henage, T. G. Walker, and M. Saffman. Fast ground state manipulation of neutral atoms in microscopic optical traps. Phys. Rev. Lett., 96(6):063001, 2006.

[20] M. Anderlini, B. L. Brown P. J. Lee and, J. Sebby-Strabley, W. D. Phillips, and J. V. Porto. 
Controlled exchange interaction between pairs of neutral atoms in an optical lattice. Nature, 448:452-456, 2007.

[21] J. Beugnon, C. Tuchendler, H. Marion, A. Gaetan, Y. Miroshnychenko, Y. R. P. Sortais, A. M. Lance, M. P. A. Jones, G. Messin, A. Browaeys, and P. Grangier. Two-dimensional transport and transfer of a single atomic qubit in optical tweezers. Nature Physics, 3:696-699, 2007.

[22] D. Hayes, P. S. Julienne, and I. H. Deutsch. Quantum logic via the exchange blockade in ultracold collisions. Phys. Rev. Lett., 98:070501, 2007.

[23] M. Lewenstein, A. Sanpera, V. Ahufinger, B. Damski, A. Sen(De), and U. Sen. Ultracold atomic gases in optical lattices: mimicking condensed matter physics and beyond. Advances in Physics, 56:243-379, 2007.

[24] K. D. Nelson, X. Li, and D. S. Weiss. Imaging single atoms in a three-dimensional array. Nature Physics, 3:556-560, 2007.

[25] S. Trotzky, P. Cheinet, S. Folling, M. Feld, U. Schnorrberger, A. M. Rey, A. Polkovnikov, E. A. Demler, M. D. Lukin, and I. Bloch. Time-resolved observation and control of superexchange interactions with ultracold atoms in optical lattices. Science, 319:295-299, 2008.

[26] A. Gaetan, Y. Miroshnychenko, T. Wilk, A. Chotia, M. Viteau, D. Comparat, P. Pillet, A. Browaeys, and P. Grangier. Observation of collective excitation of two individual atoms in the Rydberg blockade regime. Nature Physics, 5:115-118, 2009.

[27] M. Saffman, T. G. Walker, and K. Mølmer. Quantum information with Rydberg atoms. Rev. Mod. Phys., 82:2313, 2010.

[28] E. Urban, T. A. Johnson, T. Henage, L. Isenhower, D. D. Yavuz, T. G. Walker, and M. Saffman. Observation of Rydberg blockade between two atoms. Nature Physics, 5:110-114, 2009.

[29] P. Würtz, T. Langen, T. Gericke, A. Koglbauer, and H. Otto. Experimental demonstration of single-site addressability in a two-dimensional optical lattice. Phys. Rev. Lett., 103:080404, 2009.

[30] C. Deutsch, F. Ramirez-Martinez, C. Lacroûte, F. Reinhard, T. Schneider, J. N. Fuchs, F. Piéchon, F. Laloë, J. Reichel, and P. Rosenbusch. Spin self-rephasing and very long coherence times in a trapped atomic ensemble. Phys. Rev. Lett., 105:020401, 2010.

[31] A. Fuhrmanek, R. Bourgain, Y. R. P. Sortais, and A. Browaeys. Free-space lossless state detection of a single trapped atom. Phys. Rev. Lett., 106:133003, 2011.

[32] Michael J. Gibbons, Christopher D. Hamley, Chung-Yu Shih, and Michael S. Chapman. Nondestructive fluorescent state detection of single neutral atom qubits. Phys. Rev. Lett., 106:133002, 2011.

[33] L. Isenhower, E. Urban, X. L. Zhang, A. T. Gill, T. Henage, T. A. Johnson, T. G. Walker, and M. Saffman. Demonstration of a neutral atom controlled-NOT quantum gate. Phys. Rev. Lett., 104:010503, 2010.

[34] S. Olmschenk, R. Chicireanu, K. D. Nelson, and J. V. Porto. Randomized benchmarking of atomic qubits in an optical lattice. New J. Phys., 12:113007, 2010.

[35] J. F. Sherson, C. Weitenberg, M. Endres, M. Cheneau, I. Bloch, and S. Kuhr. Single-atomresolved fluorescence imaging of an atomic mott insualtor. Nature, 467:68, 2010.

[36] H. Weimer, M. Muller, Zoller P. Lesanovsky, I., and H.P. Buchler. A Rydberg quantum simulator. Nature Physics, 6:382, 2010.

[37] T. Wilk, A. Gaëtan, C. Evellin, J. Wolters, Y. Miroshnychenko, P. Grangier, and A. Browaeys. Entanglement of two individual neutral atoms using Rydberg blockade. Phys. Rev. Lett., 104:010502, 2010.

[38] X. L. Zhang, L. Isenhower, A. T. Gill, T. G. Walker, and M. Saffman. Deterministic entanglement of two neutral atoms via Rydberg blockade. Phys. Rev. A, 82:030306, 2010.

[39] A. Kay, J. K. Pachos, and C. S. Adams. Graph-state preparation and quantum computation with global addressing of optical lattices. Phys. Rev. A, 73:022310, 2006.

[40] Q. A. Turchette, C. S. Wood, B. E. King, C. J. Myatt, D. Leibfried, W. M. Itano, C. Monroe, and D. J. Wineland. Deterministic entanglement of two trapped ions. Phys. Rev. Lett., 81:3631, 
1998.

[41] J. I. Cirac and P. Zoller. A scalable quantum computer with ions in an array of microtraps. Nature, 404:579-581, 2000.

[42] C. A. Sackett, D. Kielpinski, B. E. King, C. Langer, V. Meyer, C. J. Myatt, M. Rowe, Q. A. Turchette, W. M. Itano, D. J. Wineland, and C. Monroe. Experimental entanglement of four particles. Nature, 404:256-259, 2000.

[43] F. Schmidt-Kaler, H. Hffner, M. Riebe, S. Gulde, G. P. T. Lancaster, T. Deuschle, C. Becher, C. F. Roos, J. Eschner, and R. Blatt. Realization of the Cirac-Zoller controlled-NOT quantum gate. Nature, 422:408, 2003.

[44] S. Gulde, M. Riebe, G. P. T. Lancaster, C. Becher, J. Eschner, H. Häffner, I. L. Chuang, R. Blatt, and F. Schmidt-Kaler. Implementation of the Deutsch-Jozsa algorithm on an ion-trap quantum computer. Nature, 421:48-50, 2003.

[45] D. Leibfried, B. DeMarco, V. Meyer, D. Lucas, M. Barrett, J. Britton, W. M. Itano, B. Jelenkovic, C. Langer, T. Rosenband, and D. J. Wineland. Experimental demonstration of a robust, highfidelity geometric two ion-qubit phase gate. Nature, 422:412, 2003.

[46] M. D. Barrett, J. Chiaverini, T. Schaetz, J. Britton, W. M. Itano, J. D. Jost, E. Knill, C. Langer, D. Leibfried, R. Ozeri, and D. J. Wineland. Deterministic quantum teleportation of atomic qubits. Nature, 429:737-739, 2004.

[47] J. Chiaverini, D. Leibfried, T. Schaetz, M. D. Barrett, R. B. Blakestad, J. Britton, W. M. Itano, J. D. Jost, E. Knill, C. Langer, R. Ozeri, and D. J. Wineland. Realization of quantum error correction. Nature, 432:602-605, 2004.

[48] M. Riebe, H. Häffner, C. F. Roos, W. Hansel, J. Benhelm, G. P. T. Lancaster, T. W. Korber, C. Becher, F. Schmidt-Kaler, D. F. V. James, and R. Blatt. Deterministic quantum teleportation with atoms. Nature, 429:734-737, 2004.

[49] J. Chiaverini, J. Britton, D. Leibfried, E. Knill, M. D. Barrett, R. B. Blakestad, W. M. Itano, J. D. Jost, C. Langer, R. Ozeri, T. Schaetz, and D. J. Wineland. Implementation of the semiclassical quantum fourier transform in a scalable system. Science, 308:997-1002, 2005.

[50] H. Häffner, W. Hänsel, C. F. Roos, J. Benhelm, D. Chekalkar, M. Chwalla, T. Körber, U. D. Rapol, M. Riebe, P. O. Schmidt, C. Becher, O. Gühne, W. Dür, and R. Blatt. Scalable multiparticle entanglement of trapped ions. Nature, 438:643-646, 2005.

[51] H. Häffner, F. Schmidt-Kaler, W. Haensel, C. F. Roos, T. Koerber, M. Chwalla, M. Riebe, J. Benhelm, U.D. Rapol, C. Becher, and R. Blatt. Robust entanglement. Appl. Phys. B, $81: 151,2005$.

[52] D. Leibfried, E. Knill, S. Seidelin, J. Britton, R. B. Blakestad, J. Chiaverini, D. B. Hume, W. M. Itano, J. D. Jost, C. Langer, R. Ozeri, R. Reichle, and D. J. Wineland. Creation of a six-atom Schrödinger cat state. Nature, 438:639-642, 2005.

[53] M. Riebe, K. Kim, P. Schindler, T. Monz, P. O. Schmidt, T. K. Korber, W. Hansel, H. Häffner, C. F. Roos, and R. Blatt. Process tomography of ion trap quantum gates. Phys. Rev. Lett., 97:220407, 2006.

[54] D. Stick, W. K. Hensinger, S. Olmschenk, M. J. Madsen, K. Schwab, and C. Monroe. Ion trap in a semiconductor chip. Nature Physics, 2:36-39, 2006.

[55] D. L. Moehring, P. Maunz, S. Olmschenk, K. C. Younge, D. N. Matsukevich, L.-M. Duan, and C. Monroe. Entanglement of single-atom quantum bits at a distance. Nature, 449:68-71, 2007.

[56] J. Benhelm, G. Kirchmair, C. F. Roos, and R. Blatt. Towards fault-tolerant quantum computing with trapped ions. Nature Physics, 4:463-466, 2008.

[57] D. Kielpinski. Ion-trap quantum information processing: experimental status. Frontiers of Physics in China, 3:365-381, 2008.

[58] D. Hanneke, J. P. Home, J. D. Jost, J. M. Amini, D. Leibfried, and D. J. Wineland. Realization of a programmable two-qubit quantum processor. Nature Physics, 6:13-16, 2010.

[59] A. H. Myerson, D. J. Szwer, S. C. Webster, D. T. C. Allcock, M. J. Curtis, G. Imreh, J. A. Sherman, D. N. Stacey, A. M. Steane, and D. M. Lucas. High-fidelity readout of trapped-ion 
qubits. Phys. Rev. Lett., 100:200502, 2008.

[60] T. Monz, K. Kim, W. Hansel, M. Riebe, A. S. Villar, P. Schindler, M. Chwalla, M. Hennrich, and R. Blatt. Realization of the quantum Toffoli gate with trapped ions. Phys. Rev. Lett., 102:040501, 2009.

[61] T. Monz, K. Kim, A. S. Villar, P. Schindler, M. Chwalla, M. Riebe, C. F. Roos, H. Haeffner, W. Haensel, M. Hennrich, and R. Blatt. Realization of universal ion trap quantum computation with decoherence free qubits. Phys. Rev. Lett., 103:200503, 2009.

[62] A. H. Burrell, D. J. Szwer, S. C. Webster, and D. M. Lucas. Scalable simultaneous multi-qubit readout with 99.99\% single-shot fidelity. Phys. Rev. A, 81:04030, 2010.

[63] W. C. Campbell, J. Mizrahi, Q. Quraishi, C. Senko, D. Hayes, D. Hucul, D. N. Matsukevich, P. Maunz, and C. Monroe. Ultrafast gates for single atomic qubits. Phys. Rev. Lett., 105(9):090502, 2010.

[64] T. Monz, P. Schindler, J. T. Barreiro, M. Chwalla, D. Nigg, W. A. Coish, M. Harlander, W. Haensel, M. Hennrich, and R. Blatt. 14-qubit entanglement: Creation and coherence. Phys. Rev. Lett., 106:130506, 2011.

[65] Y. Nakamura, Y. A. Pashkin, and J. S. Tsai. Coherent control of macroscopic quantum states in a single-Cooper-pair box. Nature, 398:786, 1999.

[66] Y. Nakamura, Yu. A. Pashkin, T. Yamamoto, and J. S. Tsai. Charge echo in a Cooper-pair box. Phys. Rev. Lett., 88:047901, 2002.

[67] J. M. Martinis, S. Nam, J. Aumentado, and C. Urbina. Rabi oscillations in a large Josephsonjunction qubit. Phys. Rev. Lett., 89:117901, 2002.

[68] D. Vion, A. Aassime, A. Cottet, P. Joyez, H. Pothier, C. Urbina, D. Esteve, and M. H. Devoret. Manipulating the quantum state of an electrical circuit. Science, 296:886, 2002.

[69] I. Chiorescu, Y. Nakamura, C. J. P. M. Harmans, and J. E. Mooij. Coherent quantum dynamics of a superconducting flux qubit. Science, 299:1869, 2003.

[70] A. J. Berkley, H. Xu, R. C. Ramos, M. A. Gubrud, F. W. Strauch, P. R. Johnson, J. Anderson, A. J. Dragt, C. J. Lobb, and F. C. Wellstood. Entangled macroscopic quantum states in two superconducting qubits. Science, 300:1548, 2003.

[71] T. Yamamoto, Y. A. Pashkin, O. Astafiev, Y. Nakamura, and J. S. Tsai. Demonstration of conditional gate operation using superconducting charge qubits. Nature, 425:941, 2003.

[72] A. Wallraff, D. I. Schuster, A. Blais, L. Frunzio, R. S. Huang, J. Majer, S. Kumar, S. M. Girvin, and R. J. Schoelkopf. Strong coupling of a single photon to a superconducting qubit using circuit quantum electrodynamics. Nature, 431:162, 2004.

[73] R. McDermott, R. W. Simmonds, Matthias Steffen, K. B. Cooper, K. Cicak, K. D. Osborn, Seongshik Oh, D. P. Pappas, and J. M. Martinis. Simultaneous state measurement of coupled Josephson phase qubits. Science, 307:1299-1302, 2005.

[74] T. Hime, P. A. Reichardt, B. L. T. Plourde, T. L. Robertson, C.-E. Wu, A. V. Ustinov, and John Clarke. Solid-state qubits with current-controlled coupling. Science, 314:1427-1429, 2006.

[75] M. Grajcar, A. Izmalkov, S. H. W. van der Ploeg, S. Linzen, T. Plecenik, Th. Wagner, U. Hubner, E. Il'ichev, H.-G. Meyer, A. Yu. Smirnov, Peter J. Love, Alec Maassen van den Brink, M. H. S. Amin, S. Uchaikin, and A. M. Zagoskin. Four-qubit device with mixed couplings. Physical Review Letters, 96:047006, 2006.

[76] M. Steffen, M. Ansmann, R. C. Bialczak, N. Katz, E. Lucero, R. McDermott, M. Neeley, E. M. Weig, A. N. Cleland, and J. M. Martinis. Measurement of the entanglement of two superconducting qubits via state tomography. Science, 313:1423-1425, 2006.

[77] F. Yoshihara, K. Harrabi, A. O. Niskanen, Y. Nakamura, and J. S. Tsai. Decoherence of flux qubits due to $1 / f$ flux noise. Phys. Rev. Lett., 97:167001, 2006.

[78] R. Harris, A. J. Berkley, M. W. Johnson, P. Bunyk, S. Govorkov, M. C. Thom, S. Uchaikin, A. B. Wilson, J. Chung, E. Holtham, J. D. Biamonte, A. Yu. Smirnov, M. H. S. Amin, and A. Maassen van den Brink. Sign- and magnitude-tunable coupler for superconducting flux qubits. Phys. Rev. Lett., 98:177001, 2007. 
[79] A. Lupascu, S. Saito, T. Picot, C. J. P. M. Harmans P. C. de Groot and, and J. E. Mooij. Quantum non-demolition measurement of a superconducting two-level system. Nature Physics, 3:119-125, 2007.

[80] J. Majer, J. M. Chow, J. M. Gambetta, Jens Koch, B. R. Johnson, J. A. Schreier, L. Frunzio, D. I. Schuster, A. A. Houck, A. Wallraff, A. Blais, M. H. Devoret, S. M. Girvin, and R. J. Schoelkopf. Coupling superconducting qubits via a cavity bus. Nature, 449:443-447, 2007.

[81] A. O. Niskanen, K. Harrabi, F. Yoshihara, Y. Nakamura, S. Lloyd, and J. S. Tsai. Quantum coherent tunable coupling of superconducting qubits. Science, 316:723-726, 2007.

[82] J. H. Plantenberg, P. C. de Groot, C. J. P. M. Harmans, and J. E. Mooij. Demonstration of controlled-NOT quantum gates on a pair of superconducting quantum bits. Nature, 447:836, 2007.

[83] M. A. Sillanpää, J. I. Park, and R. W. Simmonds. Coherent quantum state storage and transfer between two phase qubits via a resonant cavity. Nature, 449:438-442, 2007.

[84] T. Picot, A. Lupascu, S. Saito, C. J. P. M. Harmans, and J. E. Mooij. Role of relaxation in the quantum measurement of a superconducting qubit using a nonlinear oscillator. Phys. Rev. B, $78: 132508,2008$.

[85] J. A. Schreier, A. A. Houck, Jens Koch, D. I. Schuster, B. R. Johnson, J. M. Chow, J. M. Gambetta, J. Majer, L. Frunzio, M. H. Devoret, S. M. Girvin, and R. J. Schoelkopf. Suppressing charge noise decoherence in superconducting charge qubits. Phys. Rev. B, 77:180502, 2008.

[86] R. J. Schoelkopf and S. M. Girvin. Wiring up quantum systems. Nature, 451:664-669, 2008.

[87] M. Ansmann, H. Wang, R. C. Bialczak, M. Hofheinz, E. Lucero, M. Neeley, A. D. O'Connell, D. Sank, M. Weides, J. Wenner, A. N. Cleland, and J. M. Martinis. Violation of Bell's inequality in josephson phase qubits. Nature, 461:504, 2009.

[88] L. DiCarlo, J. M. Chow, J. M. Gambetta, L. S. Bishop, B. R. Johnson, D. I. Schuster, J. Majer, A. Blais, L. Frunzio, S. M. Girvin, and R. J. Schoelkopf. Demonstration of two-qubit algorithms with a superconducting quantum processor. Nature, 460:240-244, 2009.

[89] V. E. Manucharyan, J. Koch, L. I. Glazman, and M. H. Devoret. Fluxonium: Single Cooper-pair circuit free of charge offsets. Science, 326:113, 2009.

[90] M. Neeley, R. C. Bialczak, M. Lenander, E. Lucero, M. Mariantoni, A. D. O'Connell, D. Sank, H. Wang, M. Weides, J. Wenner, Y. Yin, T. Yamamoto, A. N. Cleland, and J. M. Martinis. Generation of three-qubit entangled states using superconducting phase qubits. Nature, 467:570, 2010.

[91] L. DiCarlo, M. D. Reed, L. Sun, B. R. Johnson, J. M. Chow, J. M. Gambetta, L. Frunzio, S. M. Girvin, M. H. Devoret, and R. J. Schoelkopf. Preparation and measurement of three-qubit entanglement in a superconducting circuit. Nature, 467:574, 2010.

[92] G. Sun, X. Wen, B. Mao, J. Chen, Y. Yu, P. Wu, and S. Han. Tunable quantum beam splitters for coherent manipulation of a solid-state tripartite qubit system. Nature Communications, $1: 51,2010$.

[93] R. Harris, M. W. Johnson, T. Lanting, A. J. Berkley, J. Johansson, P. Bunyk, E. Tolkacheva, E. Ladizinsky, N. Ladizinsky, T. Oh, F. Cioata, I. Perminov, P. Spear, C. Enderud, C. Rich, S. Uchaikin, M. C. Thom, E. M. Chapple, J. Wang, B. Wilson, M. H. S. Amin, N. Dickson, K. Karimi, B. Macready, C. J. S. Truncik, and G. Rose. Experimental investigation of an eight-qubit unit cell in a superconducting optimization processor. Phys. Rev. B, 82(2):024511, 2010.

[94] A. D. O'Connell, M. Hofheinz, M. Ansmann, R. C. Bialczak, M. Lenander, E. Lucero, M. Neeley, D. Sank, H. Wang, M. Weides, J. Wenner, J. M. Martinis, and A. N. Cleland. Quantum ground state and single-phonon control of a mechanical resonator. Nature, 464:697, 2010.

[95] A. Palacios-Laloy, F. Mallet, F. Nguyen, P. Bertet, D. Vion, D. Esteve, and A. N. Korotkov. Experimental violation of a Bells inequality in time with weak measurement. Nature Physics, $7: 442447,2010$.

[96] M. Steffen, S. Kumar, D.P. DiVincenzo, J.R. Rozen, G. A. Keefe, M. B. Rothwell, and M. B. 
Ketchen. High coherence hybrid superconducting qubit. Phys. Rev. Lett., 105:100502, 2010.

[97] M. Mariantoni. private communication, 2011.

[98] J. Bylander, S. Gustavsson, F. Yan, F. Yoshihara, K. Harrabi, G. Fitch, D. G. Cory, Y. Nakamura, J.-S. Tsai, and W. D. Oliver. Noise spectroscopy through dynamical decoupling with a superconducting flux qubit. Nature Physics, 7:565570, 2011.

[99] M. Neeley, M. Ansmann, R. C. Bialczak, M. Hofheinz, E. Lucero, A. D. O’Connell, D. Sank, H. Wang, J. Wenner, A. N. Cleland, M. R. Geller, and J. M. Martinis. Emulation of a quantum spin with a superconducting phase qudit. Science, 325:722-725, 2009.

[100] A. M. Tyryshkin, S. Tojo, J. J. L. Morton, H. Riemann, N. V. Abrosimov, P. Becker, H.-J. Pohl, T. Schenkel, M. L. W. Thewalt, K. M. Itoh, and S. A. Lyon. Electron spin coherence exceeding seconds in high purity silicon. 2011.

[101] Y. Makhlin, G. Schoen, and A. Shnirman. Quantum-state engineering with Josephson-junction devices. Rev. Mod. Phys., 73:357, 2001.

[102] J. Q. You and F. Nori. Atomic physics and quantum optics using superconducting circuits. Nature, 474:589, 2011.

[103] M. Hofheinz, H. Wang, M. Ansmann, R. C. Bialczak, E. Lucero, M. Neeley, A. D. O'Connell, D. Sank, J. Wenner, John M. Martinis, and A. N. Cleland. Synthesizing arbitrary quantum states in a superconducting resonator. Nature, 459:546-549, 2009.

[104] F. Nori. Quantum football. Science, 325:689, 2009.

[105] D. Loss and D. P. DiVincenzo. Quantum computation with quantum dots. Phys. Rev. A, $57: 120-126,1998$.

[106] A. M. Tyryshkin, S. A. Lyon, A. V. Astashkin, and A. M. Raitsimring. Electron spin relaxation times of phosphorus donors in silicon. Phys. Rev. B, 68:193207, 2003.

[107] F. Jelezko, T. Gaebel, I. Popa, A. Gruber, and J. Wrachtrup. Observation of coherent oscillations in a single electron spin. Phys. Rev. Lett., 92:076401, 2004.

[108] F. Jelezko, T. Gaebel, I. Popa, M. Domhan, A. Gruber, and J. Wrachtrup. Observation of coherent oscillation of a single nuclear spin and realization of a two-qubit conditional quantum gate. Phys. Rev. Lett., 93:130501, 2004.

[109] J. R. Petta, A. C. Johnson, J. M. Taylor, E. A. Laird, A. Yacoby, M. D. Lukin, C. M. Marcus, M. P. Hanson, and A. C. Gossard. Coherent manipulation of coupled electron spins in semiconductor quantum dots. Science, 309:2180-2184, 2005.

[110] L. Childress, M. V. Gurudev Dutt, J. M. Taylor, A. S. Zibrov, F. Jelezko, J. Wrachtrup, P. R. Hemmer, and M. D. Lukin. Coherent dynamics of coupled electron and nuclear spin qubits in diamond. Science, 314(5797):281-285, 2006.

[111] R. Hanson, F. M. Mendoza, R. J. Epstein, and D. D. Awschalom. Polarization and readout of coupled single spins in diamond. Phys. Rev. Lett., 97:087601, 2006.

[112] F. H. L. Koppens, C. Buizert, K. J. Tielrooij, I. T. Vink, K. C. Nowack, T. Meunier, L. P. Kouwenhoven, and L. M. K. Vandersypen. Driven coherent oscillations of a single electron spin in a quantum dot. Nature, 442:766-771, 2006.

[113] A. R. Stegner, C. Boehme, H. Huebl, M. Stutzmann, K. Lips, and M. S. Brandt. Electrical detection of coherent 31p spin quantum states. Nature Physics, 2:835-838, 2006.

[114] M. V. Gurudev Dutt, L. Childress, L. Jiang, E. Togan, J. Maze, F. Jelezko, A. S. Zibrov, P. R. Hemmer, and M. D. Lukin. Quantum register based on individual electronic and nuclear spin qubits in diamond. Science, 316:1312-1316, 2007.

[115] R. Hanson, L. P. Kouwenhoven, J. R. Petta, S. Tarucha, and L. M. K. Vandersypen. Spins in few-electron quantum dots. Reviews of Modern Physics, 79:1217, 2007.

[116] M. H. Mikkelsen, J. Berezovsky, L. A. Coldren N. G. Stoltz and, and D. D. Awschalom. Optically detected coherent spin dynamics of a single electron in a quantum dot. Nature Physics, 3:770$773,2007$.

[117] K. C. Nowack, F. H. L. Koppens, Y. V. Nazarov, and L. M. K. Vandersypen. Coherent control of a single electron spin with electric fields. Science, 318:1430-1433, 2007. 
[118] X. Xu, B. Sun, P. R. Berman, D. G. Steel, A. S. Bracker, D. Gammon, and L. J. Sham. Coherent optical spectroscopy of a strongly driven quantum dot. Science, 317:929-932, 2007.

[119] S. Amasha, K. MacLean, I. P. Radu, D. M. Zumbuhl, M. A. Kastner, M. P. Hanson, and A. C. Gossard. Electrical control of spin relaxation in a quantum dot. Phys. Rev. Lett., 100:046803, 2008.

[120] J. Berezovsky, M. H. Mikkelsen, N. G. Stoltz, L. A. Coldren, and D. D. Awschalom. Picosecond coherent optical manipulation of a single electron spin in a quantum dot. Science, 320:349-352, 2008.

[121] L. Chirolli and G. Burkard. Decoherence in solid state qubits. Advances in Physics, 57:225, 2008.

[122] B. D. Gerardot, D. Brunner, P. A. Dalgarno, P. Ohberg, S. Seidl, M. Kroner, K. Karrai, N. G. Stoltz, P. M. Petroff, and R. J. Warburton. Optical pumping of a single hole spin in a quantum dot. Nature, 451:441-444, 2008.

[123] P. Neumann, N. Mizuochi, F. Rempp, P. Hemmer, H. Watanabe, S. Yamasaki, V. Jacques, T. Gaebel, F. Jelezko, and J. Wrachtrup. Multipartite entanglement among single spins in diamond. Science, 320:1326-1329, 2008.

[124] G. Balasubramanian, P. Neumann, D. Twitchen, M. Markham, R. Kolesov, N. Mizuochi, J. Isoya, J. Achard, J. Beck, J. Tissler, V. Jacques, P.R. Hemmer, F. Jelezko, and J. Wrachtrup. Ultralong spin coherence time in isotopically engineered diamond. Nature Materials, 8:383-387, 2009.

[125] C. Barthel, D. J. Reilly, C. M. Marcus, M. P. Hanson, and A. C. Gossard. Rapid single-shot measurement of a singlet-triplet qubit. Phys. Rev. Lett., 103:160503, 2009.

[126] S. Foletti, H. Bluhm, D. Mahalu, V. Umansky, and A. Yacoby. Universal quantum control of two-electron spin quantum bits using dynamic nuclear polarization. Nature Physics, 5:903, 2009.

[127] J. Twamley and S. D. Barrett. A superconducting cavity bus for single Nitrogen Vacancy defect centres in diamond. Phys. Rev. B, 81:241202, 2010.

[128] C. Barthel, J. Medford, C. M. Marcus, M. P. Hanson, and A. C. Gossard. Interlaced dynamical decoupling and coherent operation of a singlet-triplet qubit. Phys. Rev. Lett., 105(26):266808, 2010.

[129] H. Bluhm, S. Foletti, D. Mahalu, V. Umansky, and A. Yacoby. Enhancing the coherence of a spin qubit by operating it as a feedback loop that controls its nuclear spin bath. Phys. Rev. Lett., 105:216803, 2010.

[130] G. de Lange, Z. H. Wang, D. Rist, V. V. Dobrovitski, and R. Hanson. Universal dynamical decoupling of a single solid-state spin from a spin bath. Science, 330:60, 2010.

[131] S. Nadj-Perge, S. M. Frolov, E. P. A. M. Bakkers, and L. P. Kouwenhoven. Spin-orbit qubit in a semiconductor nanowire. Nature, 468:1084, 2010.

[132] H. Bluhm, S. Foletti, I. Neder, M. Rudner, D. Mahalu, V. Umansky, and A. Yacoby. Dephasing time of GaAs electron-spin qubits coupled to a nuclear bath exceeding $200 \mu \mathrm{s}$. Nature Physics, $7: 109,2011$.

[133] A. Morello, J. J. Pla, F. A. Zwanenburg, K. W. Chan, K. Y. Tan, H. Huebl, M. Mottonen, C. D. Nugroho, C. Yang, J. A. van Donkelaar, A. D. C. Alves, D. N. Jamieson, C. C. Escott, L. C. L. Hollenberg, R. G. Clark, and A. S. Dzurak. Single-shot readout of an electron spin in silicon. Nature, 467:687, 2010.

[134] D. R. McCamey, J. Van Tol, G. W. Morley, and C. Boehme. Electronic spin storage in an electrically readable nuclear spin memory with a lifetime $>100$ seconds. Science, 330:1652$1656,2010$.

[135] W. M. Witzel, M. S. Carroll, A. Morello, L. Cywiński, and S. Das Sarma. Electron spin decoherence in isotope-enriched silicon. Phys. Rev. Lett., 105:187602, 2010.

[136] C. B. Simmons, J. R. Prance, B. J. Van Bael, Teck Seng Koh, Zhan Shi, D. E. Savage, M. G. Lagally, R. Joynt, Mark Friesen, S. N. Coppersmith, and M. A. Eriksson. Tunable spin loading 
and $t 1$ of a silicon spin qubit measured by single-shot readout. Phys. Rev. Lett., 106:156804, 2011.

[137] S. Simmons, R. M. Brown, H. Riemann, N. V. Abrosimov, P. Becker, H. J. Pohl, L. W. Thewalt, and J. J. L. Morton. Entanglement in a solid-state spin ensemble. Nature, 470:6972, 2011.

[138] D. Suter and T. S. Mahesh. Spins as qubits: Quantum information processing by nuclear magnetic resonance. J. Chem. Phys., 128:052206, 2008.

[139] X. Peng, Z. Liao, N. Xu, G. Qin, X. Zhou, D. Suter, and J. Du. Quantum adiabatic algorithm for factorization and its experimental implementation. Phys. Rev. Lett., 101:220405, 2008.

[140] C. Negrevergne, T. S. Mahesh, C. A. Ryan, M. Ditty, F. Cyr-Racine, W. Power, N. Boulant, T. Havel, D. G. Cory, and R. Laflamme. Benchmarking quantum control methods on a 12qubit system. Phys. Rev. Lett., 96:170501, 2006.

[141] L. M. K. Vandersypen, M. Steffen, G. Breyta, C. S. Yannoni, M. H. Sherwood, and I. L. Chuang. Experimental realization of Shor's quantum factoring algorithm using nuclear magnetic resonance. Nature, 414:883-887, 2001.

[142] M. Grajcar, S. H. W. van der Ploeg, A. Izmalkov, E. Il'ichev, H.-G. Meyer, A. Fedorov, A. Shnirman, and Gerd Schon. Sisyphus cooling and amplification by a superconducting qubit. Nature Physics, 4:612-616, 2008.

[143] F. Nori. Atomic physics with a circuit. Nature Physics, 4:589, 2008.

[144] C. Langer, R. Ozeri, J. D. Jost, J. Chiaverini, B. DeMarco, A. Ben-Kish, R. B. Blakestad, J. Britton, D. B. Hume, W. M. Itano, D. Leibfried, R. Reichle, T. Rosenband, T. Schaetz, P. O. Schmidt, and D. J. Wineland. Long-lived qubit memory using atomic ions. Phys. Rev. Lett., 95:060502, 2005.

[145] H. J. Kimble. The quantum internet. Nature, 453:1023-1030, 2008.

[146] Y. Colombe, T. Steinmetz, G. Dubois, F. Linke, D. Hunger, and J. Reichel. Strong atom-field coupling for Bose-Einstein condensates in an optical cavity on a chip. Nature, 450:272-276, 2007.

[147] P. F. Herskind, A. Dantan, J. P. Marler, M. Albert, and M. Drewsen. Realization of collective strong coupling with ion Coulomb crystals in an optical cavity. Nature Physics, 5:494-498, 2009.

[148] D. Englund, A. Faraon, I. Fushman, N. Stoltz, P. Petroff, and J. Vuckovic. Controlling cavity reflectivity with a single quantum dot. Nature, 450:857-861, 2007.

[149] J. L. O'Brien. Optical quantum computing. Science, 318:1567-1570, 2007.

[150] L. Tian, P. Rabl, R. Blatt, and P. Zoller. Interfacing quantum-optical and solid-state qubits. Phys. Rev. Lett., 92:247902, 2004.

[151] L. Tian, R. Blatt, and P. Zoller. Scalable ion trap quantum computing without moving ions. Eur. Phys. J. D, 32:201-208, 2005.

[152] J. Verdu, H. Zoubi, Ch. Koller, J. Majer, H. Ritsch, and J. Schmiedmayer. Strong magnetic coupling of an ultracold gas to a superconducting waveguide cavity. Phys. Rev. Lett., 103:043603, 2009.

[153] D. Petrosyan, G. Bensky, G. Kurizki, I. Mazets, J. Majer, and J. Schmiedmayer. Reversible state transfer between superconducting qubits and atomic ensembles. Phys. Rev. A, 79:040304, 2009.

[154] C. Zipkes, S. Palzer, C. Sias, and M. Kohl. A trapped single ion inside a Bose-Einstein condensate. Nature, 464:388, 2010.

[155] H. Doerk, Z. Idziaszek, and T. Calarco. Atom-ion quantum gate. Phys. Rev. A, 81:012708, 2010.

[156] Y. Kubo, F. R. Ong, P. Bertet, D. Vion, V. Jacques, D. Zheng, A. Dréau, J.-F. Roch, A. Auffeves, F. Jelezko, J. Wrachtrup, M. F. Barthe, P. Bergonzo, and D. Esteve. Strong coupling of a spin ensemble to a superconducting resonator. Phys. Rev. Lett., 105:140502, 2010.

[157] D. I. Schuster, A. P. Sears, E. Ginossar, L. DiCarlo, L. Frunzio, J. J. L. Morton, H. Wu, G. A. D. Briggs, B. B. Buckley, D. D. Awschalom, and R. J. Schoelkopf. High-cooperativity coupling of electron-spin ensembles to superconducting cavities. Phys. Rev. Lett., 105:140501, 2010.

[158] H. Wu, R. E. George, J. H. Wesenberg, K. Mølmer, D. I. Schuster, R. J. Schoelkopf, K. M. Itoh, 
A. Ardavan, J. J. L. Morton, and G. A. D. Briggs. Storage of multiple coherent microwave excitations in an electron spin ensemble. Phys. Rev. Lett., 105:140503, 2010.

[159] C. Simon, M. Afzelius, J. Appel, A. Boyer de la Giroday, S. J. Dewhurst, N. Gisin, C. Y. Hu, F. Jelezko, S. Kroll, J. H. Muller, J. Nunn, E. Polzik, J. Rarity, H. de Riedmatten, W. Rosenfeld, A. J. Shields, N. Skold, R. M. Stevenson, R. Thew, I. Walmsley, M. Weber, H. Weinfurter, J. Wrachtrup, and R. J. Young. Quantum memories. a review based on the european integrated project qubit applications (qap). Eur. Phys. J. D, 58:1, 2010.

[160] M. Wallquist, K. Hammerer, P. Rabl, M. Lukin, and P. Zoller. Hybrid quantum devices and quantum engineering. Physica Scripta, T137:014001, 2009.

[161] D. P. DiVincenzo. Quantum computation. Science, 270:255-261, 1995.

[162] I. M. Buluta and F. Nori. Quantum simulators. Science, 326:108 - 111, 2009.

[163] Z. Kim, B. Suri, V. Zaretskey, S. Novikov, K. D. Osborn, A. Mizel, F. C. Wellstood, and B. S. Palmer. Decoupling a cooper-pair box to enhance the lifetime to $0.2 \mathrm{~ms}$. Phys. Rev. Lett., 106:120501, 2011.

[164] J. I. Cirac and P. Zoller. Quantum computations with cold trapped ions. Phys. Rev. Lett., 74:4091, 1995.

[165] K. Mølmer and A. Sørensen. Multiparticle entanglement of hot trapped ions. Phys. Rev. Lett., $82: 1835,1999$. 